\title{
Orbit classification in exoplanetary systems
}

\author{
Euaggelos E. Zotos ${ }^{1}$, Bálint Érdi ${ }^{2}$, Tareq Saeed ${ }^{3}$, and Mohammed Sh. Alhodaly ${ }^{3}$ \\ ${ }^{1}$ Department of Physics, School of Science, Aristotle University of Thessaloniki, 54124 Thessaloniki, Greece \\ e-mail: evzotos@physics.auth.gr \\ 2 Department of Astronomy, Eötvös University, Pázmány Péter sétány 1/A, 1117 Budapest, Hungary \\ e-mail: B.Erdi@astro.elte.hu \\ ${ }^{3}$ Nonlinear Analysis and Applied Mathematics (NAAM)-Research Group, Department of Mathematics, Faculty of Science, \\ King Abdulaziz University, PO Box 80203, Jeddah 21589, Saudi Arabia \\ e-mail: tsalmalki@kau.edu.sa
}

Received 30 November 2019 / Accepted 12 January 2020

\begin{abstract}
The circular version of the restricted three-body problem, along with the method of grid classification are used to determine the character of the trajectories of a test particle, which move in a binary exoplanetary system. The binary system can be either a parent star-exoplanet or an exoplanet-exoplanet or exomoon, while the test particle is considered to be an asteroid or comet, a space probe, or even a small exomoon in the case where the primary body is a star. By using modern two-dimensional color maps, we succeeded in classifying the starting conditions and distinguishing between bounded, escaping, and collision type of motion for the test particle. Furthermore, in the case of bounded regular motion, we further classify the starting conditions by considering their geometry (revolving around one or both main bodies) and orientation (prograde or retrograde, with respect to a rotating coordinate system of the primaries). For the initial setup of the test particle we consider two starting conditions: the launch from pericenter or apocenter. The final states are qualitatively visualized through two-dimensional basin diagrams. This approach allowed us to systematically investigate and extract dynamical information on the dependency of the test particle final state as a function of the particle's initial semi-major axis and eccentricity for a given primary and secondary mass ratio. Finally, we applied the restricted three-body model on several exoplanetary systems with observed mass-ratios and studied the dynamical behavior of a test-mass.
\end{abstract}

Key words. methods: numerical - celestial mechanics

\section{Introduction}

There is no doubt that nowadays the topic of exoplanets is one of the most active and interesting fields in Astronomy. According to NASA Exoplanet Archive ${ }^{1}$, there are more than 4100 confirmed exoplanets in 3067 exosolar systems, of which 671 have more than one exoplanet. Therefore, these new findings strongly suggest that our Solar System should not be considered as a typical planetary system, but more as an exception. Consequently, the intervals regarding the typical numerical values of the orbital eccentricity, the semi-major axis, as well as the mass ratio of the planetary systems should be revised.

It is observed for many exoplanets that the corresponding masses are very similar to the masses of the planets of our Solar System. The main difference is that some exoplanets follow high eccentric orbits, while they circulate relatively close to their parent stars. The majority of the exoplanets move on planar circular orbits, with an orbital period of 8-12 days, while they have a Jovian mass value. On the other hand, only a small minority of exoplanets follow trajectories with high eccentricity and/or inclination (e.g., HD 43197b, HD 45350b, HD 80606b, HD 96167b, GJ 317c, etc.). Interestingly, in the case where multiple exoplanets exist in an exosolar system, they appear to be locked in mean-motion resonances (MMRs). In particular, a significant fraction of them move in 2:1 resonance (e.g., HD 73456 , HD 82943, HD 128311), while the other types of resonances are also possible that is, 3:1 (e.g., HD 60532), 3:2 (e.g., HD 45364), 4:1 (e.g., HD 108874), 4:3, and 5:2 (see e.g., Antoniadou 2016;

\footnotetext{
1 https://exoplanetarchive.ipac.caltech.edu/
}

Campanella 2011; Campanella et al. 2013; Correia et al. 2010; Couetdic et al. 2010; Laskar \& Correia 2004; Goździewski et al. 2006; Lovis et al. 2011; Rein et al. 2010).

The circular version of the restricted three-body problem is very useful, in its simplicity, for describing the motion in exoplanetary systems. For the case where the distance between the two bodies is large enough, that is outside the Hill's sphere of influence we have the one-dimensional models (1D), which were developed to describe the averaged dynamics in the coplanar case (see e.g., Érdi 1977; Robutel \& Pousse 2013). Obviously, in the scenario that involves eccentric and/or inclined motion, the orbital dynamics in phase space is much more complicated due to the higher number of degrees of freedom. For example, in the eccentric case, additional coorbital configurations emerge (see e.g., Mikkola et al. 2006; Namouni 1999; Pousse et al. 2017; Sidorenko et al. 2014), while in the inclined case we have new retrograde coorbital configurations (Morais \& Namouni 2013). The coorbital model has been applied in many studies to search for Trojan exoplanets (see e.g., Érdi \& Sándor 2005; Funk et al. 2012; Hippke \& Angerhausen 2015; Laughlin \& Chambers 2002; Leleu et al. 2017; Schwarz et al. 2012).

In recent years, several studies have been devoted to demystifying the dynamics of exoplanetary systems by using various dynamical models and numerical techniques (see e.g., Antoniadou 2016; Antoniadou \& Voyatzis 2014, 2016; FerrazMello et al. 2006; Hadjidemetriou 2006; Henrard \& Libert 2008; Lee 2004; Michtchenko et al. 2006; Voyatzis 2008). Most of these works attempt to either distinguish between order and chaos, or to present a new numerical technique to detect 
nonlinear dynamics or study specific resonant configurations considering families of periodic orbits. Conversely, in our work we apply the computational methods introduced and applied in Nagler $(2004,2005)$ in order to perform a thorough and systematic taxonomy of the staring conditions of the test particle. Following this technique allows us to gain insight into different types of phase-space basins (corresponding to bounded, escaping, and collision motion) on two-dimensional maps. For the starting conditions of the massless particle, the approach used in Érdi et al. (2012) is adopted, thus considering only pericentric and apocentric launching.

The article is organized according to the following layout: Sect. 2 contains the mathematical formulation of the dynamical system, followed by Sect. 3, where we describe the computational methodology, used for the taxonomy of the trajectories. The main outcomes of our analysis are analyzed in Sect. 4 and our paper ends with the main conclusions given in Sect. 5 .

\section{Mathematical formulation of the dynamical system}

In our analysis, we shall use the model of the restricted threebody problem, according to which a (massless) test particle moves under the gravitational influence of two bodies with considerably larger masses. These two bodies $B_{1}$ and $B_{2}$ (with masses $m_{1}$ and $m_{2}$ ), known as the primary and secondary respectively, move on a planar and circular trajectory around their common center of gravity. It should be noted, that the plane of motion of $B_{1}$ and $B_{2}$ coincides with that of the massless particle $m$. Then, the on-plane motion of the test particle is governed, according to the equations of motion formulated in a co-rotating reference frame (see e.g., Murray \& Dermott 1999)

$\ddot{x}-2 \dot{y}=\frac{\partial \Phi}{\partial x}, \quad \ddot{y}+2 \dot{x}=\frac{\partial \Phi}{\partial y}$,

where

$\Phi(x, y)=\frac{m_{1}}{\sqrt{\left(x-x_{1}\right)^{2}+y^{2}}}+\frac{m_{2}}{\sqrt{\left(x-x_{2}\right)^{2}+y^{2}}}+\frac{1}{2}\left(x^{2}+y^{2}\right)$

is the negative effective potential, and where $x_{1}=-\mu$ and $x_{2}=$ $1-\mu$ are the positions of two main bodies on the $x$-axis. In addition, $m_{1}=1-\mu$ and $m_{2}=\mu$, where $\mu=m_{2} /\left(m_{1}+m_{2}\right) \leq 1 / 2$ is the mass parameter.

For monitoring the motion of the test particle we use Cartesian coordinates $(x, y)$, with a frame of reference which rotates along with the primaries $B_{1}$ and $B_{2}$, while its origin coincides with the center of mass of the two main bodies (see Fig. 1). Moreover, the constant of gravity $G$, the distance between $B_{1}$ and $B_{2}(R)$, as well as the sum of the masses are equal to unity ( $\left.G=R=m_{1}+m_{2}=1\right)$. Therefore, in a fixed system of coordinates, the bodies $B_{1}$ and $B_{2}$ perform one full revolution in $2 \pi$ time units.

The dynamical system's corresponding Hamiltonian reads

$H(x, y, \dot{x}, \dot{y})=-\left(\dot{x}^{2}+\dot{y}^{2}\right)+2 \Phi(x, y)=C_{0}$,

where $C_{0}$ denotes the Jacobi constant which is related to the orbital energy as $C_{0}=-2 E_{0}$.

\section{Description of the computational methods}

In order to determine the character of the motion of the massless particle, we integrate large sets of $1024 \times 1024$ starting

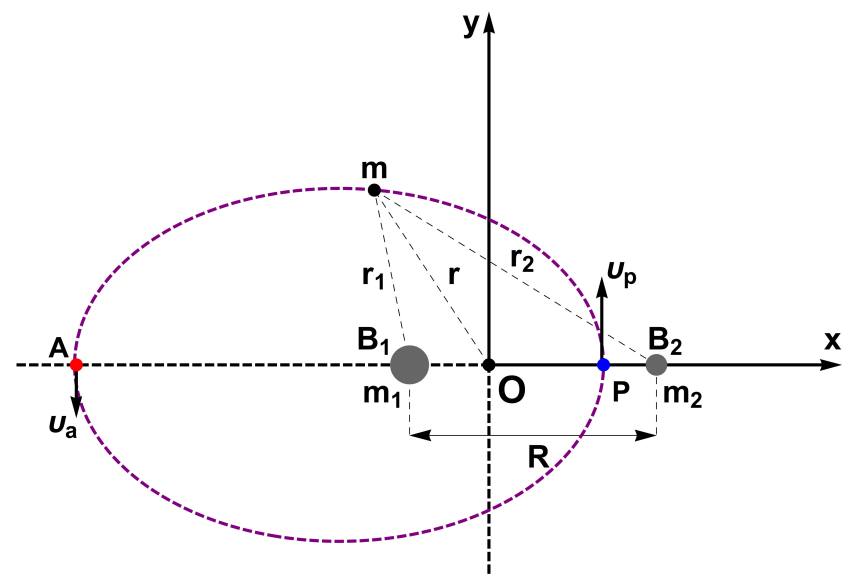

Fig. 1. Planar configuration of the two main bodies $B_{1}$ and $B_{2}$, along with the massless particle $m$, which is launched either from its pericenter $P$, with velocity $v_{\mathrm{p}}$, or from its apocenter $A$, with velocity $v_{\mathrm{a}}$. In both cases, the vector of the initial velocity is perpendicular to the horizontal $x$-axis. $O$ is the mass center of the bodies $B_{1}$ and $B_{2}$.

conditions in several types of two-dimensional maps. In all cases, the test particle is launched from the $x$-axis, either from the pericenter or from the apocenter of its initial assumed elliptic orbit. Moreover, the velocity is perpendicular to the $x$-axis in the direction of rotation of the primaries, that is, the direction of the rotation of the rotating system. Specifically, in the case of pericentric launching we have

$x_{0}=\alpha(1-e)-\mu, \quad \dot{y_{0}}=\sqrt{\frac{1-\mu}{\alpha} \frac{1+e}{1-e}}-\alpha(1-e)$,

where in the case of the apocentric launching we have

$x_{0}=-\alpha(1+e)-\mu, \quad \dot{y_{0}}=-\sqrt{\frac{1-\mu}{\alpha} \frac{1-e}{1+e}}+\alpha(1+e)$,

while in both cases we take $y_{0}=\dot{x_{0}}=0$, thus following the approach used in Érdi et al. (2012).

The parameters $e$ and $\alpha$ correspond to the eccentricity and semi-major axis of the test particle's initial eccentric orbit, respectively. However, later on, the trajectory of the massless particle can be altered to some other type.

For the final state, regarding the type of motion of the test particle, we have three main categories: (i) trajectories that remain bounded and circulate around one or both main bodies, (ii) trajectories that lead to a collision with either the primary or the secondary, and (iii) trajectories that escape from the gravitational attraction of the binary system.

For the case of bounded trajectories, the motion of the test particle can be further classified as: (i) non-escaping regular, (ii) trapped sticky, and (ii) trapped chaotic, by using one of the available chaos indicators. In our study, we choose to apply the Smaller Alignment Index (SALI; Skokos 2001).

In the case of regular bounded motion, the test particle can circulate around one or both main bodies $B_{1}$ and $B_{2}$, while its orientation can be clockwise (retrograde) or counterclockwise (prograde or direct). The distinction between prograde and retrograde motion can be computationally achieved by simply obtaining the initial value of the angular momentum $L_{z 0}$. At this point, we should clarify that the well-known equation $L_{z}=$ $x \dot{y}-y \dot{x}$ is valid only for trajectories that circulate around both or between the two main bodies, that is when the reference point 

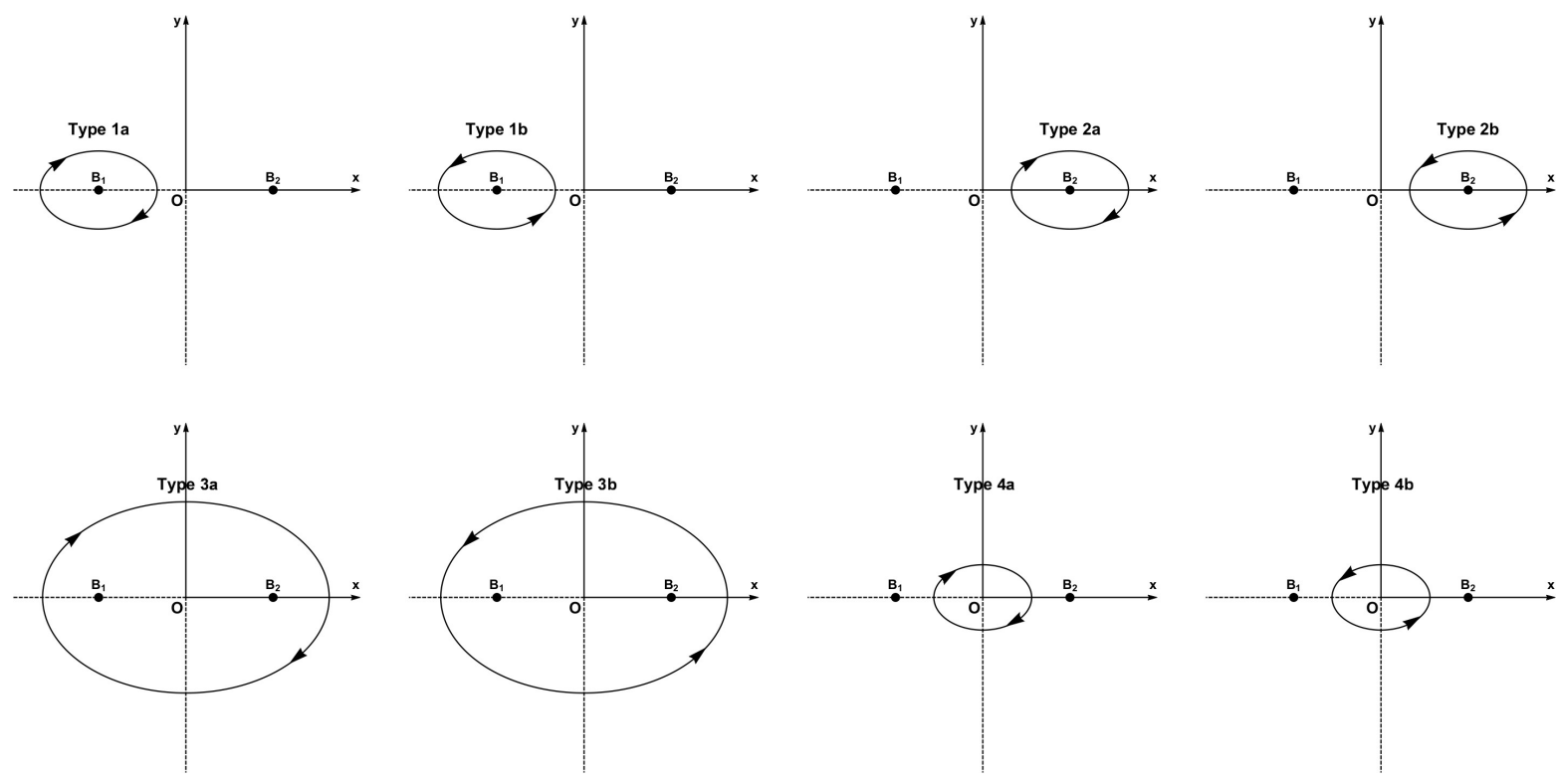

Fig. 2. Schematics illustrating the eight possible configurations of the bounded regular orbits.

regarding the direction of movement is the origin O. However, when the massless particle moves around one of the bodies $B_{1}$ or $B_{2}$, the equation of the angular momentum should be modified as $L_{z}=(x+\mu) \dot{y}-y \dot{x}$ and $L_{z}=(x+\mu-1) \dot{y}-y \dot{x}$, respectively. In other words, the reference point, regarding the orientation of the movement, should be transferred from the origin $\mathrm{O}$ to the respective centers of the bodies $B_{1}$ and $B_{2}$.

In order to determine whether an ordered orbit circulates around one or both main bodies during the numerical integration, we computed the minimum $\left(x_{\min }\right)$ and the maximum $\left(x_{\max }\right)$ values of the coordinate $x$. Then, following the approach used in Nagler (2004, 2005), the regular trajectories can be further classified in the following eight sub-classes:

1. Type 1a: if $x_{\min }<x_{1}, x_{\max }<x_{2}$ and $L_{z 0}<0$ then the trajectory circulates around the primary $B_{1}$, in a retrograde direction.

2. Type 1b: if $x_{\min }<x_{1}, x_{\max }<x_{2}$ and $L_{z 0}>0$ then the trajectory circulates around the primary $B_{1}$, in a prograde direction.

3. Type 2a: if $x_{\min }>x_{1}, x_{\max }>x_{2}$ and $L_{z 0}<0$ then the trajectory circulates around the secondary $B_{2}$, in a retrograde direction.

4. Type 2b: if $x_{\min }>x_{1}, x_{\max }>x_{2}$ and $L_{z 0}>0$ then the trajectory circulates around the secondary $B_{2}$, in a prograde direction.

5. Type 3a: if $x_{\min }<x_{1}, x_{\max }>x_{2}$ and $L_{z 0}<0$ then the trajectory circulates around both bodies $B_{1}$ and $B_{2}$, in a retrograde direction.

6. Type 3b: if $x_{\min }<x_{1}, x_{\max }>x_{2}$ and $L_{z 0}>0$ then the trajectory circulates around both bodies $B_{1}$ and $B_{2}$, in a prograde direction.

7. Type 4a: if $x_{\min }>x_{1}, x_{\max }<x_{2}$ and $L_{z 0}<0$ then the trajectory circulates between both bodies $B_{1}$ and $B_{2}$, in a retrograde direction.

8. Type 4b: if $x_{\min }>x_{1}, x_{\max }<x_{2}$ and $L_{z 0}>0$ then the trajectory circulates between both bodies $B_{1}$ and $B_{2}$, in a prograde direction.

The schematics in Fig. 2 visualize the eight main possible types of regular orbits. Needless to say that the above sub-classes apply not only to periodic trajectories but also to quasi-periodic ones.
Also, it is necessary to mention, that all the above-mentioned types of motion refer to the rotating system, thus the retrograde types are retrograde in the rotating system, but prograde in a fixed system, because the launch is in the direction of rotation.

A trajectory is considered to escape when the test particle moves far away from the system of the two bodies and therefore their gravitational attraction becomes infinitesimal. The threshold value for escape is set to $r=\sqrt{x^{2}+y^{2}}>50$. On the other hand, the massless particle leads to a collision with either the primary $B_{1}$ or the secondary $B_{2}$, when $r<10^{-5}$.

The equations of motion (Eq. (1)) were numerically integrated by using a variable time step Bulirsch-Stoer integrator, while the corresponding routine was written in FORTRAN 77 (Press et al. 1992). The total time of the numerical integration was set to $10^{4}$ time units $\left(10^{4} / 2 \pi\right.$ revolutions), so as to ensure that all trajectories have enough time to unveil their true character. Throughout the computations, the conservation of the Jacobi constant $C$ was sufficient enough, taking into account that the corresponding error was of the order of $10^{-14}$. The average required computation time, per color map, was varying between $10 \mathrm{~h}$ and 5 days, using a Quad-Core i7 4.0 GHz CPU (without using an MPI code), with 32 GB of RAM. The latest version 12.0 of Mathematica ${ }^{\circledR}$ (Wolfram 2003) was utilized for the graphics of the article.

\section{Orbit taxonomy in binary systems}

The system contains three free parameters, that is the mass parameter $\mu$, as well as the semi-major axis $\alpha$ along with the eccentricity $e$ of the initial eccentric orbit of the test particle.

\subsection{The $(\alpha, e)$ survey}

We begin with maps on the $(\alpha, e)$-plane for given values of $\mu$. After retrieving from NASA Exoplanet Archive the updated data regarding the confirmed exoplanets, we choose those binary systems of parent star-exoplanet, in which the two main bodies move in circular orbits (so as to be able to use the circular version of the restricted three-body problem). 
(a)

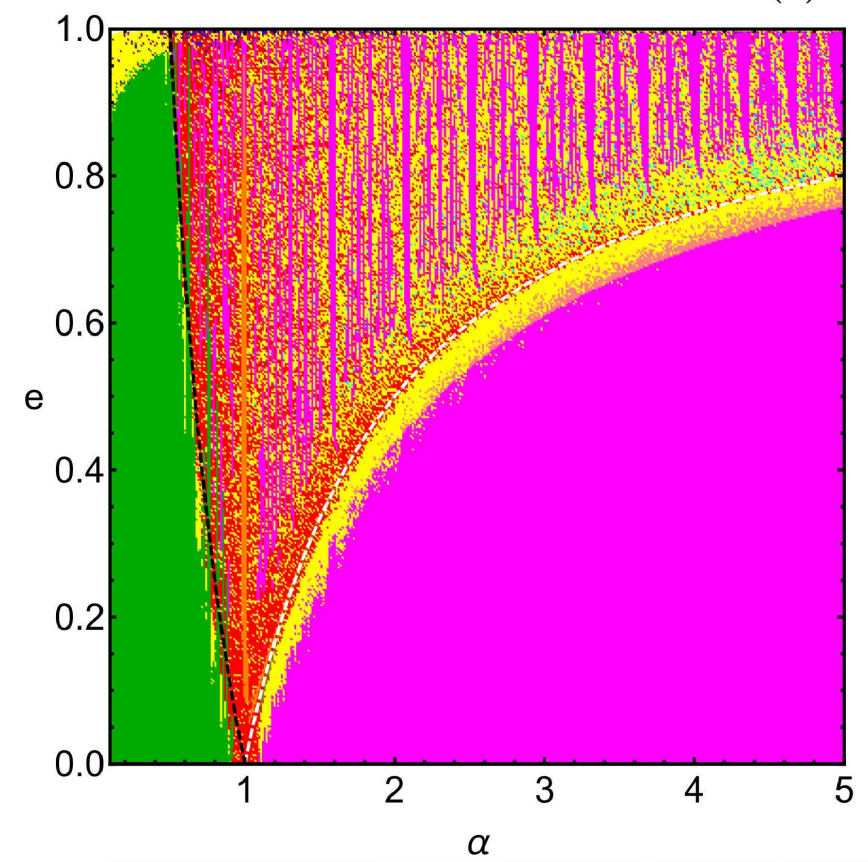

(b)

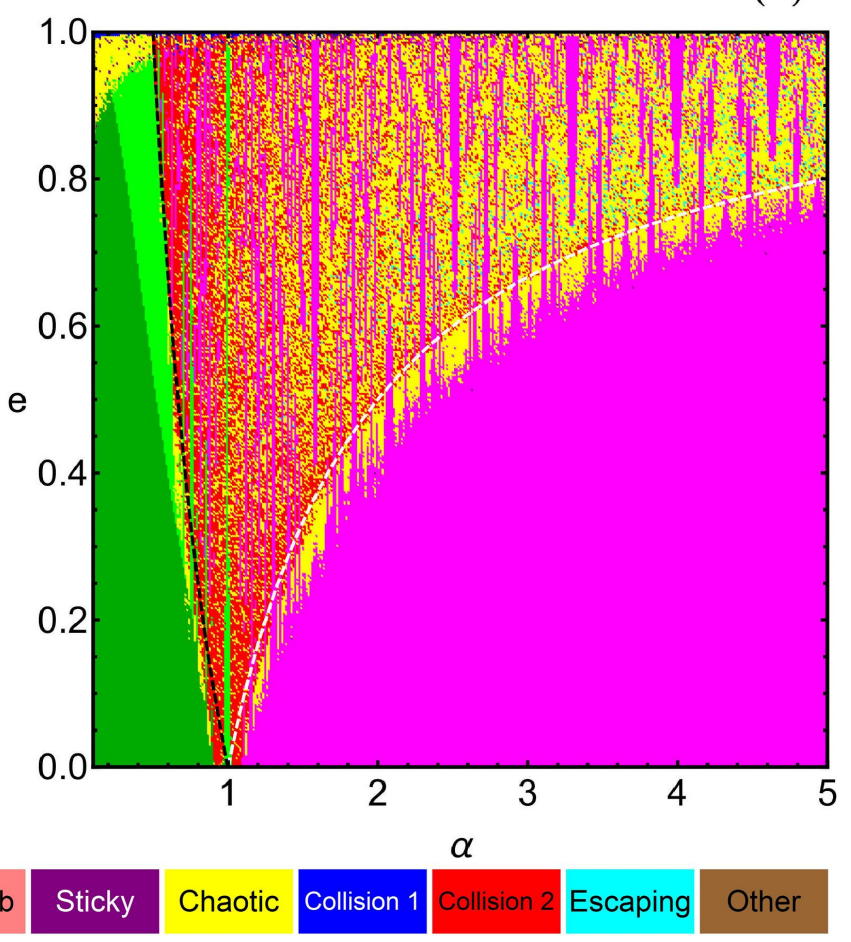

Fig. 3. Color diagrams showing the basins on the $(\alpha, e)$-plane, for $\mu=0.0001$, when the test particle is launched from its $(a)$ pericenter, and (b) apocenter. The black dashed line marks the position where the apocenter distance is $\alpha(1+e)=1$, while the white dashed line indicates the position where the pericenter distance is $\alpha(1-e)=1$.

In Figs. $3 \mathrm{a}$ and $\mathrm{b}$ we present the basin diagrams on the $(\alpha, e)$-plane, when $\mu=0.0001$. This value of the mass parameter corresponds to cases in which the exoplanet has a significantly smaller mass than the corresponding parent star. According to the so far obtained data, the exoplanets CoRoT-24 c and HD $103197 \mathrm{~b}$, along with their parent stars, fit this particular value of the mass parameter. The test mass then takes the role of an additional planet or an exomoon around either CoRoT-24c or HD103197b, respectively. Part a of Fig. 3 corresponds to trajectories starting from the pericenter, while part $b$ of the same figure contains trajectories launched from the apocenter of the initial eccentric orbit of the massless particle. In these diagrams, the black dashed line indicates the position where the apocentric distance is $d_{\mathrm{a}}=\alpha(1+e)=1$, while the white dashed line marks the position where $d_{\mathrm{p}}=\alpha(1-e)=1$, that is when the pericenter of the massless particle reaches the orbit of the secondary (exoplanet).

We observe, that in both cases (launching from the pericenter or apocenter), when $d_{\mathrm{a}}<1$ the motion of the test particle is bounded. Specifically, the massless particle mainly moves in bounded regular trajectories around the primary (parent star). Moreover, when it is launched from its pericenter the motion is performed always in a prograde orientation (Type 1b), while when it is launched from its apocenter retrograde motion around the primary (Type 1a), is also possible. It should be noted, that for relatively high values of the eccentricity $(e>0.9)$ the chaotic and sticky motion dominates (see the upper left corners of the diagrams). The $d_{\mathrm{a}}>1$ part of the diagrams is divided into two main regions, where $d_{\mathrm{p}}>1$ and $d_{\mathrm{p}}<1$. The $d_{\mathrm{p}}>1$ area is covered by a unified basin, corresponding to simple loop trajectories that circulate around both the parent star and the exoplanet, while the $d_{\mathrm{p}}<1$ area contains numerous islands of resonant orbits that also circulate around both main bodies of the system.
All types of motion around $B_{1}$ and $B_{2}$ are retrograde (Type 3a), while there is no numerical indication of prograde motion around them (Type $3 \mathrm{~b}$ ). Inside the $d_{\mathrm{p}}<1$ region one can identify some small islands of starting conditions, corresponding to Type 2 a motion, that is retrograde motion around the exoplanet, while prograde motion around the secondary seems to be absent.

Around the line $d_{\mathrm{p}}=1$ we see a mixture of starting conditions that correspond either to chaotic/sticky motion or to collision with the exoplanet. It is interesting to note that for such small values of the mass parameter, escaping trajectories, as well as trajectories leading to a collision with the parent star (primary), are extremely limited. Indeed, our computations suggest that both types of motion are possible, however, the corresponding starting conditions appear only as isolated points, scattered in the $d_{\mathrm{p}}<1$ area, without forming basins of escape or collision.

It should be noted, that in all studied cases, we encountered a small portion of sticky orbits. However, the corresponding starting conditions appear only as lonely points (not forming regions) and they are always deeply embedded inside the chaotic yellow regions of the color-coded maps. Therefore they are not quite visible.

In Fig. 4 we provide the distribution of the values of the Jacobi constant, for the starting conditions of part a of Fig. 3 . We see, that for relatively low values of the semi-major axis $(\alpha<0.4)$ we measured the highest values of $C$, while the lowest values of the Jacobi constant correspond to starting conditions with $e>0$.9. It is interesting to note how the values of $C$ spike when $d_{\mathrm{p}}=1$. This happens because when $d_{\mathrm{p}}=\alpha(1-e)=1$ the second term in the effective potential $\Phi(x, y)$ becomes singular. Exactly along the curve $d_{\mathrm{p}}=1$ the initial conditions lead to an immediate collision with the secondary and therefore the numerical integration is not possible for them. This curve acts, in a way, as an additional forbidden region. However, initial conditions 


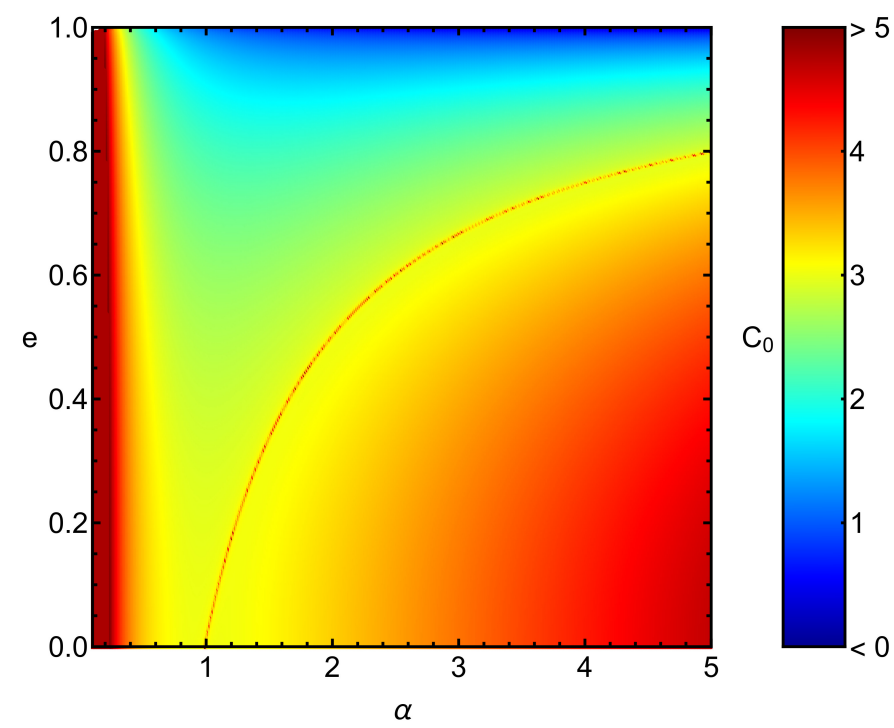

Fig. 4. Distribution of the values of the Jacobi constant, for the starting conditions, shown in part a of Fig. 3. The red curve corresponds to $d_{p}=1$.

outside this line (from both sides) can be numerically integrated and the respective values of the Jacobi constant are relatively high, since we are in the near vicinity of a "forbidden region".

According to the data from NASA Exoplanet Archive, for the majority of the exoplanetary systems, that can be modeled by the restricted three-body problem, the value of the mass parameter is about $\mu=0.001$. In Table 1 we provide the details of 33 exoplanetary systems with compatible values of $\mu$. We note that in most of the cases presented in Table 1 the exoplanet is the sole exoplanet in the respective exosolar system.

In Figs. 5a and b we depict the basin diagrams on the $(\alpha, e)$ plane, for $\mu=0.001$. For the regions with $d_{\mathrm{a}}<1$ the orbital structure is quite similar to that we have seen earlier in Fig. 3. Moreover, we can now clearly see the stability islands of the resonant periodic orbits, located in the $d_{\mathrm{p}}<1$ region. Figure 6 shows ten characteristic resonant periodic orbits, with starting conditions from part a of Fig. 5. One can see, that initially, the trajectory circulates around the exoplanet (see part a), while with increasing value of the semi-major axis, the trajectory evolves around both bodies (star and exoplanet), while at the same time it becomes much more complicated (in other words its multiplicity increases). The positions of the ten periodic solutions on the $(\alpha, e)$ plane are shown in part a of Fig. 5, using black five-pointed stars.

When the trajectories are launched from the pericenter (see part a of Fig. 5) around the line $d_{\mathrm{p}}=1$ we can see the stability islands corresponding to both retrograde (Type $2 a$ ) and prograde (Type 2b) motion around the exoplanet. The first type of stability islands is located just above the curve $d_{\mathrm{p}}=1$, while the second type of stability islands can be seen just below the same curve. In addition, in part b of Fig. 5 we observe that the two regions $d_{\mathrm{p}}<$ 1 and $d_{\mathrm{p}}>1$ are somehow connected, through the continuation of stability islands, corresponding to Type 3a motion. When $\mu=$ 0.001 , the presence of escaping trajectories, in the $d_{\mathrm{p}}<1$ region, is stronger, while also collision orbits to the primary are present, mainly for $e>0.8$.

The next value of the mass parameter under consideration is $\mu=0.01$, while characteristic examples of exoplanets, with compatible values of $\mu$, are the NN Ser (AB) c and RR Cae b. Figs. 7a and $\mathrm{b}$ displays the basin structure on the $(\alpha, e)$-plane, for
Table 1. Data of confirmed exoplanets: mass of the exoplanet $m_{\mathrm{p}}$ (in Jupiter masses $M_{\mathrm{J}}$ ), mass of the parent star $m_{\mathrm{s}}$ (in solar masses $M_{\odot}$ ), and the corresponding value of the mass parameter $\mu$.

\begin{tabular}{lccc}
\hline \hline Exoplanet name & $m_{\mathrm{p}}\left(M_{\mathrm{J}}\right)$ & $m_{\mathrm{s}}\left(M_{\odot}\right)$ & $\mu$ \\
\hline CoRot-1 b & 1.03 & 0.95 & 0.001083 \\
HAT-P-5 b & 1.06 & 1.16 & 0.000910 \\
HAT-P-8 b & 1.34 & 1.28 & 0.001045 \\
HAT-P-49 b & 2.20 & 2.22 & 0.000990 \\
HATS-34 b & 0.94 & 0.95 & 0.000984 \\
HATS-35 b & 1.22 & 1.32 & 0.000927 \\
HD 330075 b & 0.48 & 0.47 & 0.001020 \\
K2-137 b & 0.50 & 0.46 & 0.001085 \\
K2-237 b & 1.24 & 1.23 & 0.001000 \\
K2-260 b & 1.42 & 1.39 & 0.001020 \\
KELT-3 b & 1.94 & 1.96 & 0.000988 \\
KELT-14 b & 1.29 & 1.24 & 0.001034 \\
KELT-23 b & 0.94 & 0.94 & 0.000997 \\
Kepler-11 g ${ }^{* *}$ & 0.95 & 0.95 & 0.000999 \\
Kepler-539 b ${ }^{(*)}$ & 0.97 & 1.05 & 0.000924 \\
ksi Aql b & 2.02 & 2.20 & 0.000917 \\
Lupus-TR-3 b & 0.81 & 0.87 & 0.000931 \\
OGLE-TR-132 b & 1.17 & 1.26 & 0.000928 \\
OGLE-TR-182 b & 1.06 & 1.14 & 0.000929 \\
TrES-2 b & 1.49 & 1.36 & 0.001094 \\
WASP-2 b & 0.84 & 0.84 & 0.001007 \\
WASP-12 b & 1.47 & 1.43 & 0.001024 \\
WASP-24 b & 1.07 & 1.13 & 0.000947 \\
WASP-26 b & 1.03 & 1.12 & 0.000917 \\
WASP-41 b ${ }^{(*)}$ & 0.85 & 0.81 & 0.001048 \\
WASP-44 b & 0.89 & 0.95 & 0.000933 \\
WASP-58 b & 0.89 & 0.94 & 0.000945 \\
WASP-75 b & 1.07 & 1.14 & 0.000937 \\
WASP-95 b & 1.13 & 2.20 & 0.000917 \\
WASP-108 b & 1.17 & 1.16 & 0.000999 \\
WASP-112 b & 0.88 & 0.81 & 0.001089 \\
WASP-122 b & 1.40 & 1.41 & 0.000999 \\
XO-1 b & 0.83 & 0.88 & 0.000942 \\
\hline & & & \\
\hline
\end{tabular}

Notes. The asterisk ${ }^{(*)}$, at the names of the exoplanets, indicates that there exist more than one exoplanet in the respective exosolar system.

$\mu=0.01$. The most prominent aspects of the orbital dynamics of the test particle are the following: (i) the stability islands of the resonant orbits in the $d_{\mathrm{p}}<1$ area are reduced, especially in the case where the test particle is launched from its apocenter, (ii) the area of the stability islands, corresponding to Type $2 \mathrm{a}$ and $2 \mathrm{~b}$ trajectories around the exoplanet (which exist around the curve $d_{\mathrm{p}}=1$ ) increases, (iii) retrograde regular motion (Type 1a) around the primary is possible only in the case of a launch from the apocenter, (iv) regular motion (both prograde and retrograde) around the exoplanet is possible only when the massless particle is launched from its pericenter, and (v) basins composed of starting conditions leading to a collision with the exoplanet mainly exist when the test particle is launched from its pericenter, while in the case that it is launched from its apocenter the same type of collision trajectories form significantly smaller basins of collision.

From the catalog of the exoplanets, the highest possible value of the mass parameter is $\mu=0.1875$ and it corresponds to the exoplanetary system $2 \mathrm{M} 2206-20$. The respective character of motion of the test particle on the $(\alpha, e)$-plane is given in Figs. $8 \mathrm{a}$ 
(a)

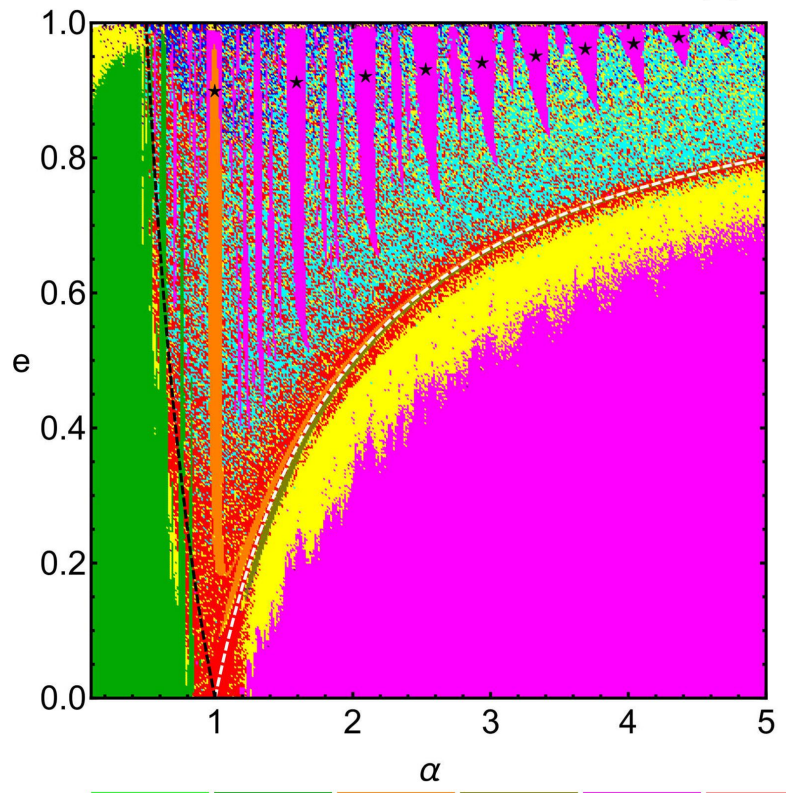

(b)

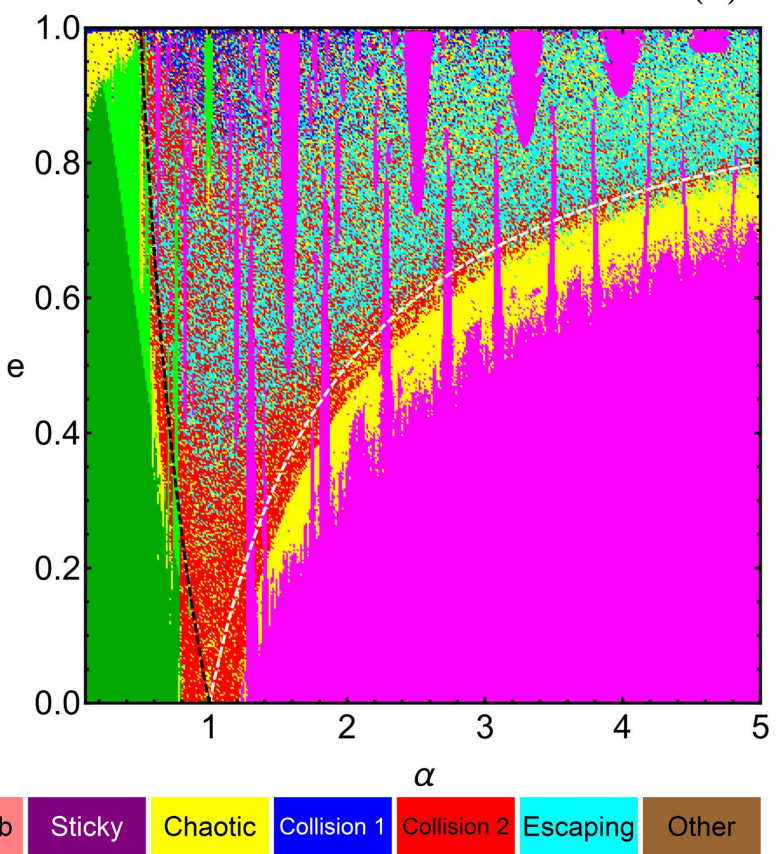

Fig. 5. Color diagrams showing the basins on the $(\alpha, e)$-plane, for $\mu=0.001$, when the test particle is launched from its $(a)$ pericenter and (b) apocenter. The black dashed line marks the position where the apocenter distance is $\alpha(1+e)=1$, while the white dashed line indicates the position where the pericenter distance is $\alpha(1-e)=1$. The black five-pointed stars indicate the position of the periodic orbits of Fig. 6 .

(a)

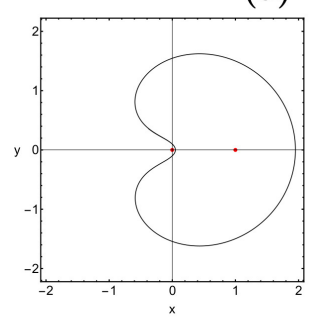

(f)

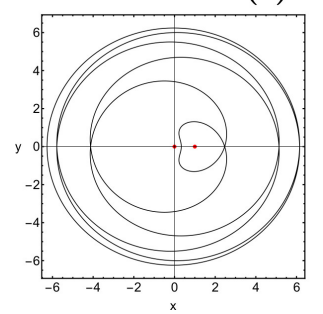

(b)

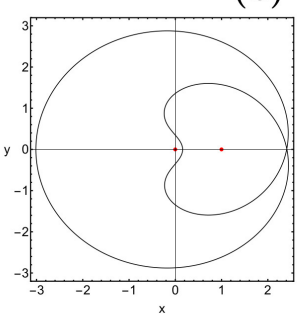

(g)

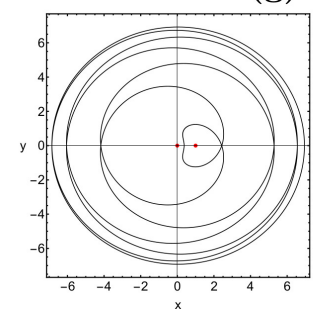

(c)

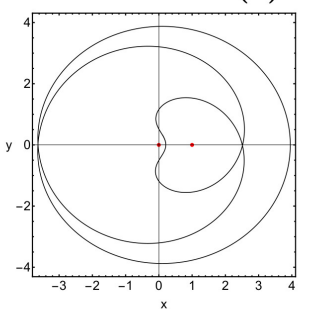

(h)

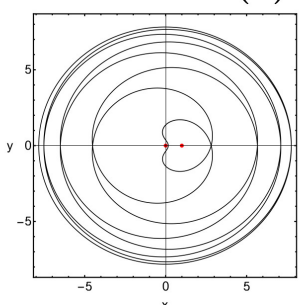

(d)

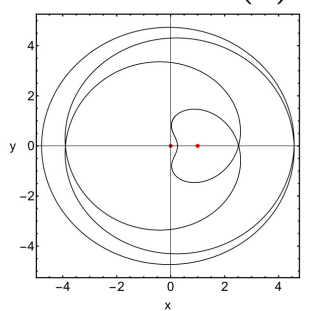

(i)

(e)
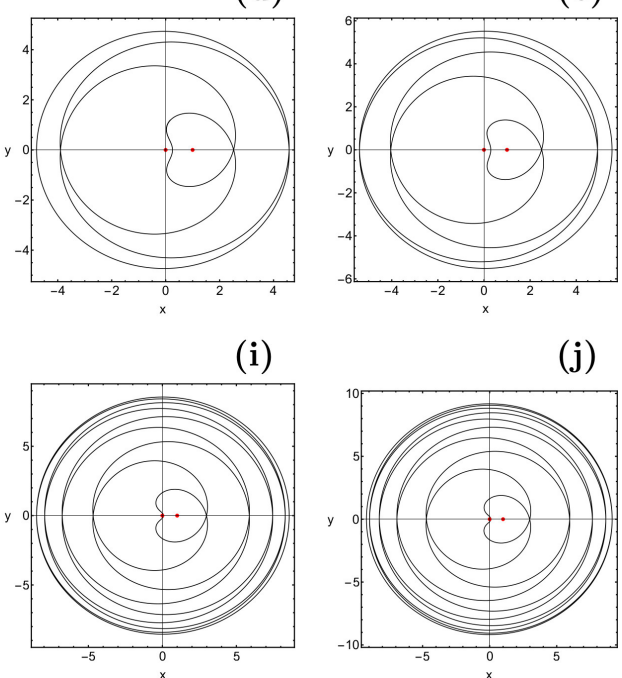

(j)

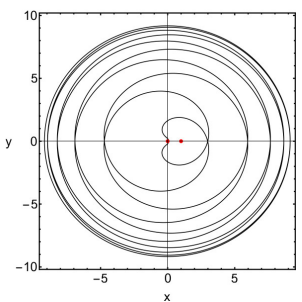

Fig. 6. Sample characteristic resonant periodic orbits, when $\mu=0.001$, with starting conditions from part a of Fig. 5 . The red dots pinpoint the locations of the two bodies (star and exoplanet).

and $b$. Here, it is evident that the basin structure has many differences, with respect to the previously studied cases. In the case where the test particle is launched from its pericenter (see part a), first of all, one can observe the complete absence of trajectories (simple or of higher resonance) that revolve around both main bodies (Type $3 \mathrm{a})$. In fact, the $d_{\mathrm{p}}>1$ part of the $(\alpha, e)$-plane is now covered by a complicated mixture of escaping and collision trajectories. In the $d_{\mathrm{p}}<1$ part of the same plane, there is still one main stability island of retrograde trajectories around the exoplanet, but there is no evidence of prograde motion around the secondary. Furthermore, it should be noted that for this value of the mass parameter, it is the first time that starting conditions that lead to a collision with the primary (star) form substantial basins of collision.

In the case that the massless particle is launched from its apocenter (see part b of Fig. 8) retrograde regular motion around both bodies (Type 3a) dominates the $(\alpha, e)$-plane. Specifically, the corresponding stability island crosses the curve $d_{\mathrm{p}}=1$ and covers both regions with $d_{\mathrm{p}}<1$ and $d_{\mathrm{p}}>1$. Another interesting aspect is the fact that the retrograde motion around the primary (Type 1a) is extremely confined, while a small stability island is present in the $d_{\mathrm{a}}<1$ area and for relatively high values of the eccentricity $(e>0.8)$. Moreover, one can see, that in this case, the basin boundaries of the stability island of Type 3a motion 
(a)

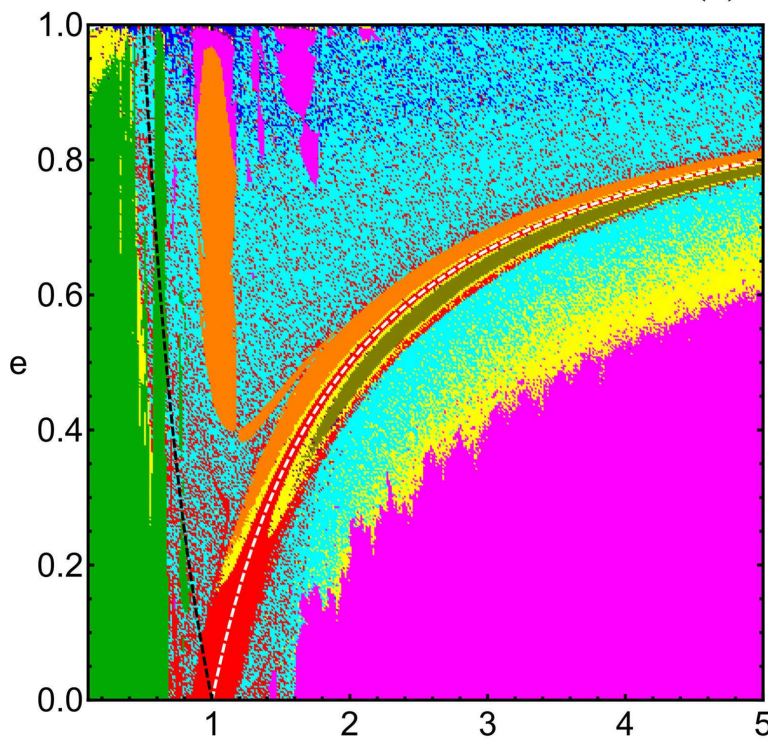

$\alpha$

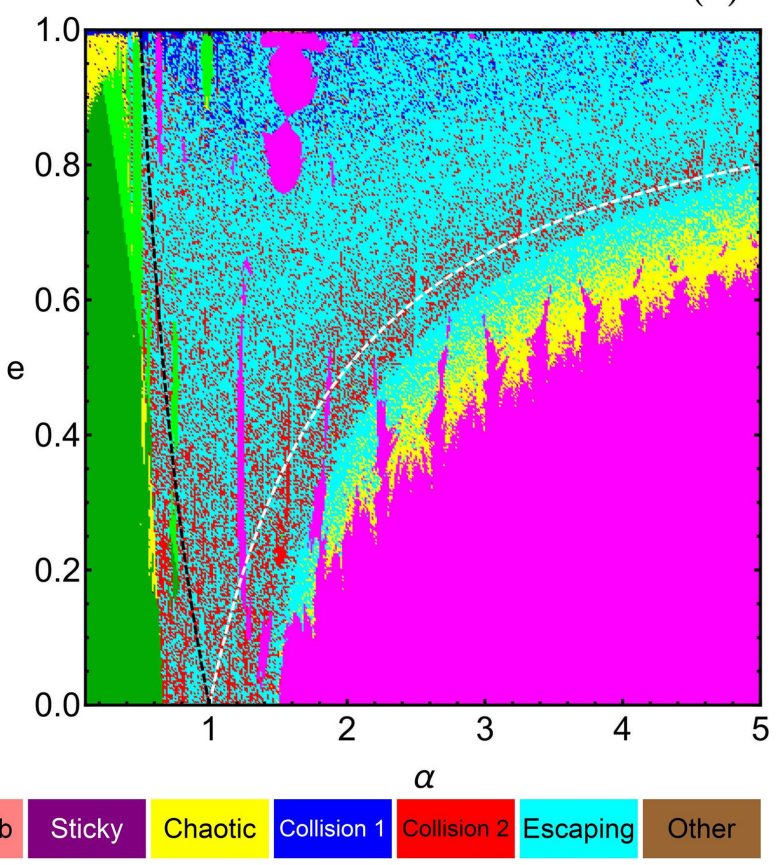

Fig. 7. Color diagrams showing the basins on the $(\alpha, e)$-plane, for $\mu=0.01$, when the test particle is launched from its $(a)$ pericenter and $(b)$ from its apocenter. The black dashed line marks the position where the apocenter distance is $\alpha(1+e)=1$, while the white dashed line indicates the position where the pericenter distance is $\alpha(1-e)=1$.

(a)

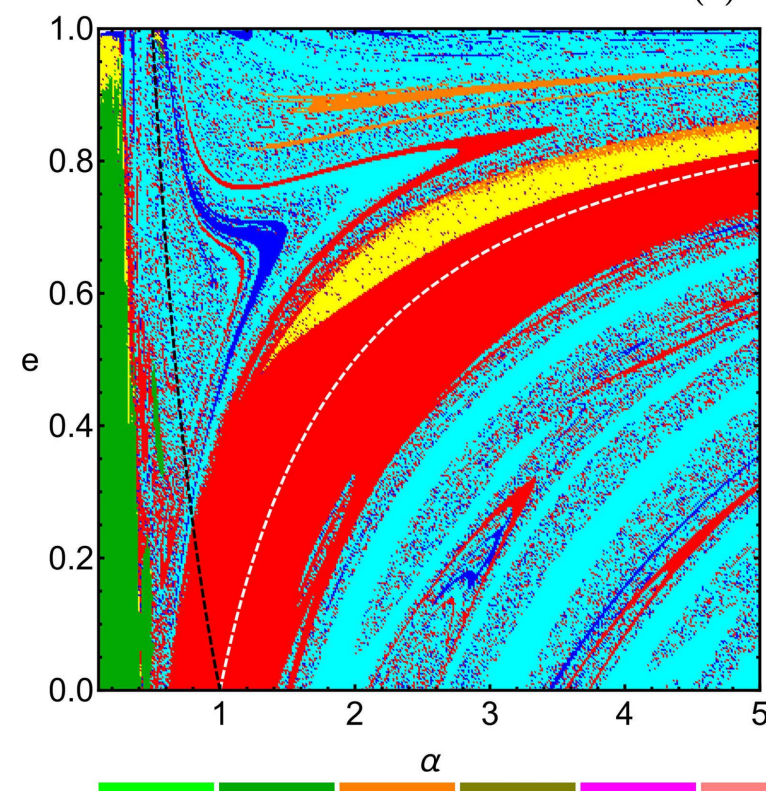

a)

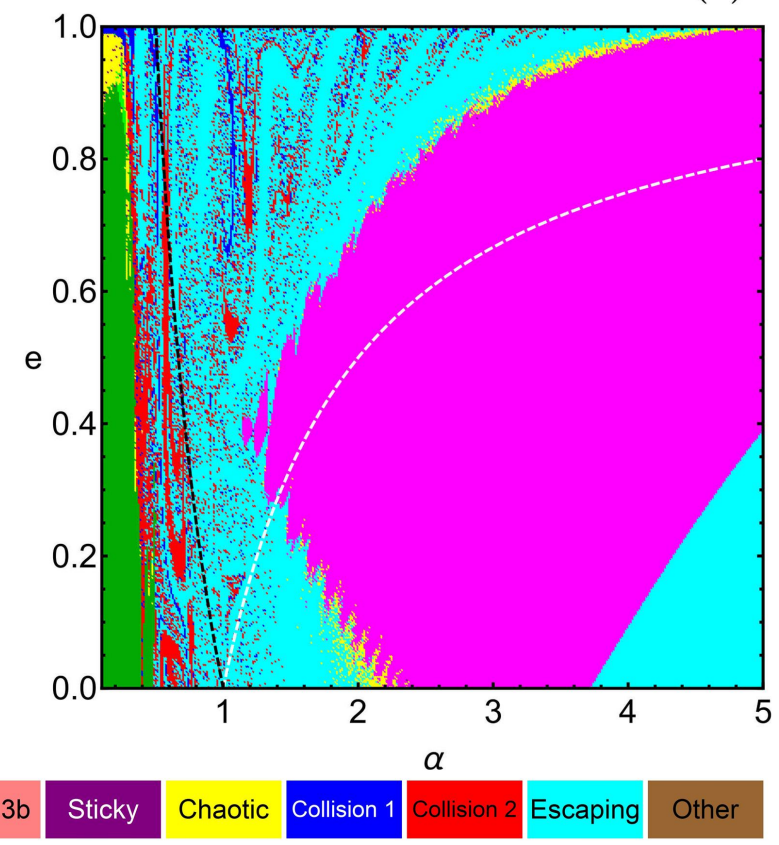

Fig. 8. Color diagrams showing the basins on the $(\alpha, e)$-plane, for $\mu=0.1875$, when the test particle is launched from its $(a)$ pericenter and (b) apocenter. The black dashed line marks the position where the apocenter distance is $\alpha(1+e)=1$, while the white dashed line indicates the position where the pericenter distance is $\alpha(1-e)=1$.

are more smooth. In particular, in all previous cases we have seen that in the vicinity of the basin boundaries of the stability islands of Type 3 a motion, there was a considerable amount of starting conditions corresponding to trapped chaotic motion. However, in this case, it is seen that the rate of chaotic motion is much lower.

Figures $9 \mathrm{a}$ and $\mathrm{b}$ shows the character of motion of the massless particle, for $\mu=0.4$. Of course, such high values of the mass parameter are not possible in a binary system of starexoplanet, but they are possible in other types of exoplanetary systems, such as exoplanet-exoplanet or even exoplanet-exomoon (see e.g., Teachey \& Kipping 2018). In such cases, the role of the test particle could be played by an asteroid/comet or a space probe. With respect to the previous case, the orbital content on the $(\alpha, e)$-plane has several major differences. In the case 
(a)

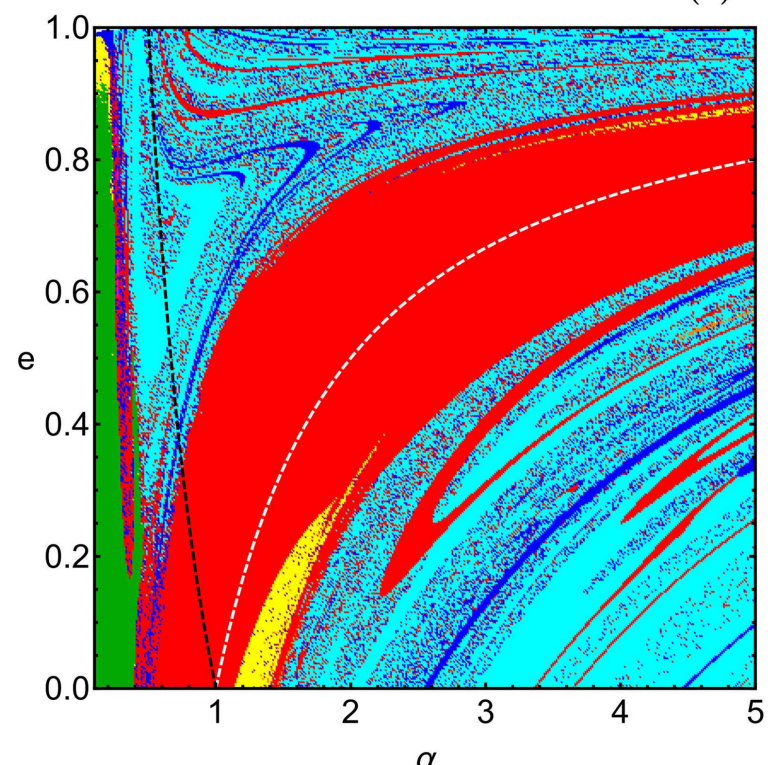

(b)

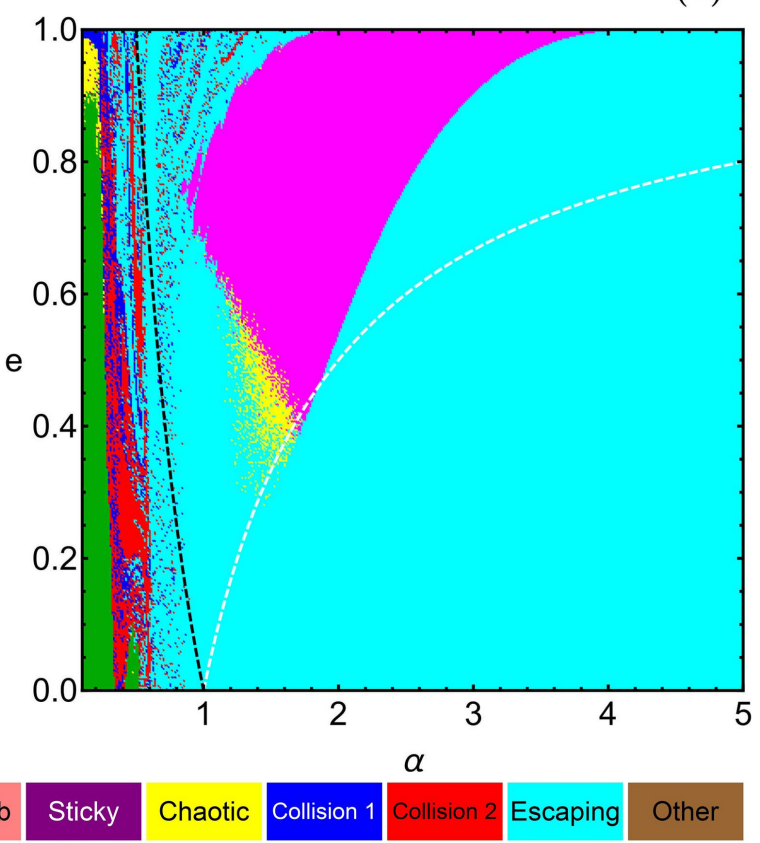

Fig. 9. Color diagrams showing the basins on the $(\alpha, e)$-plane, for $\mu=0.4$, when the test particle is launched from its $(a)$ pericenter and (b) apocenter. The black dashed line marks the position where the apocenter distance is $\alpha(1+e)=1$, while the white dashed line indicates the position where the pericenter distance is $\alpha(1-e)=1$.

of launching the test particle from its pericenter (see part a of Fig. 9) regular bounded motion around the exoplanet is no longer dynamically possible, while the area of the collision basin to secondary has been increased. Moreover, the area of the basin corresponding to regular prograde motion around the primary has been further confined. Part b of Fig. 9 shows the final states of the test particle when it has been launched from the apocenter. Now escaping motion is the dominant type of motion (especially in the $d_{\mathrm{p}}>1$ region), while at the same time, the stability island which contains the starting conditions of simple retrograde trajectories around both bodies (Type 3a) has been almost completely transferred to the $d_{\mathrm{p}}<1$ region of the $(\alpha, e)$-plane.

The pericentric velocity of the test particle decreases when the value of $\alpha$ increases. Thus, for $\alpha<1$ the test particle is launched with larger velocity, than the circular velocity of the secondary. Therefore, in the rotating system, the orbit of the test particle is direct (meaning the direction of rotation). However, for $\alpha>1$ the test particle is launched with smaller velocity than the circular velocity of the secondary and thus the test particle moves slower than the primaries. Viewing this from the rotating system, the test particle moves backward, in a retrograde direction. The above analysis explains why for $\alpha<1$ we see a strong presence of Type $1 \mathrm{~b}$ motion and also why for $\alpha>1$ the dominant type of regular motion is Type 3a. Moreover, to obtain Type $3 \mathrm{~b}$ trajectories, the test particle must be started perpendicularly to the $x$-axis but in the opposite direction of the rotation of the primary.

\subsection{The $(\alpha, \mu)$ survey}

So far, we studied the character of motion with starting conditions on the $(\alpha, e)$-plane, for specific values of the mass parameter $\mu$. Now, we will provide results on the $(\alpha, \mu)$-plane, for specific values of the eccentricity $e$. Figures $10 \mathrm{a}-\mathrm{f}$ shows the orbital structure of the $(\alpha, \mu)$-plane when the test particle is launched from its pericenter. In this type of $2 \mathrm{D}$ color map, we can clearly distinguish how the mass parameter influences the motion of the test particle. One can observe the following phenomena: (i) retrograde motion around both main bodies (Type 3a) exists only for $\mu<0.1$ and also when $e<0.6$, (ii) prograde motion around the primary is almost always possible, for relatively low values of the semi-major axis $(\alpha<0.5)$. However, for extremely high values of the eccentricity (e.g., when $e=0.95$ ) the regular motion turns to chaotic, (iii) prograde and retrograde motion around the exoplanet is possible mainly for $\mu<0.2$, while the corresponding stability islands are situated around the line $d_{\mathrm{p}}=1$, as it can be seen on parts c and d of Fig. 10, (iv) around both sides of the line $d_{\mathrm{p}}=1$ we see the presence of a basin composed of starting conditions leading to a collision with the exoplanet. With increasing value of the eccentricity the size of this basin grows, while at the same time its location moves to higher values of the semi-major axis, and (v) the remaining area of the $(\alpha, \mu)$-plane is covered by a mixture of starting conditions that form basins of escape and collision with highly complicated shapes.

In Figs. 11a-f we present the basin diagrams on the $(\alpha, \mu)$ plane when the test particle is launched from its apocenter. In this case, we see that the changes in the orbital structure are milder, with respect to what we have seen in the previous case when the test particle is launched from its pericenter. As the value of the eccentricity increases, the most important change concerns the stability island of the Type 3a trajectories. Specifically, the stability island moves on higher values of $\mu$, with an increasing value of $e$. Moreover, the same stability island divides the $(\alpha, \mu)$-plane into two regions: (i) the region above the island, which is completely dominated by escaping trajectories and (ii) the region below the stability islands, which contains a rich mixture of starting conditions, corresponding to both escaping and collision trajectories. 
(a)

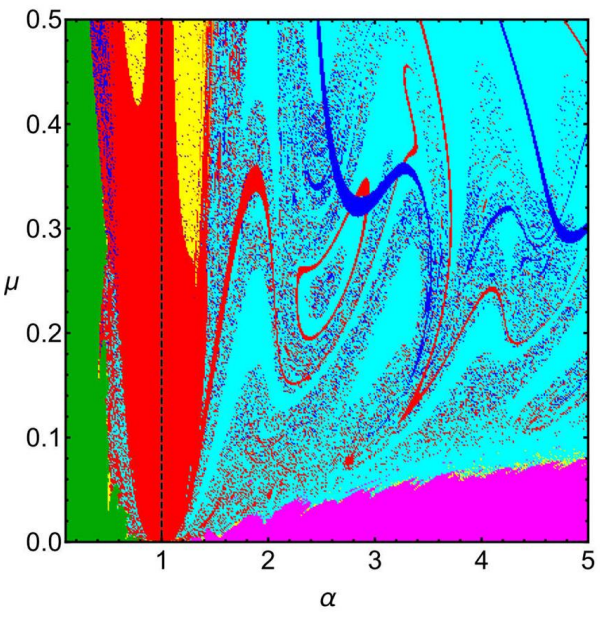

(c)

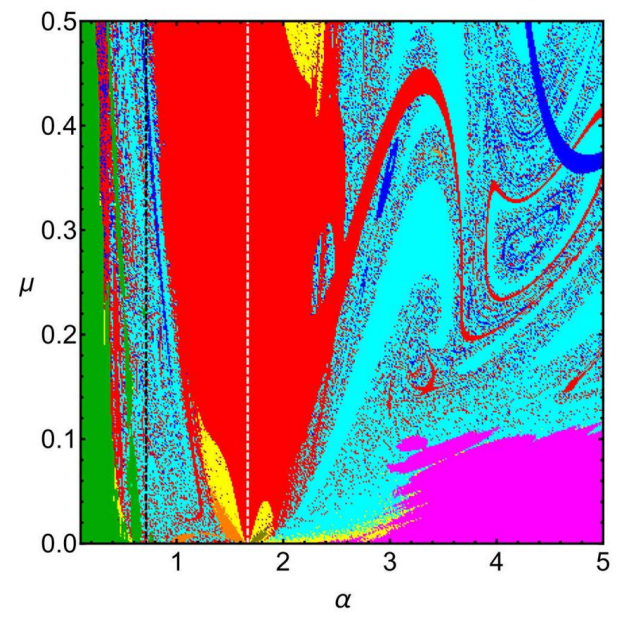

(e)

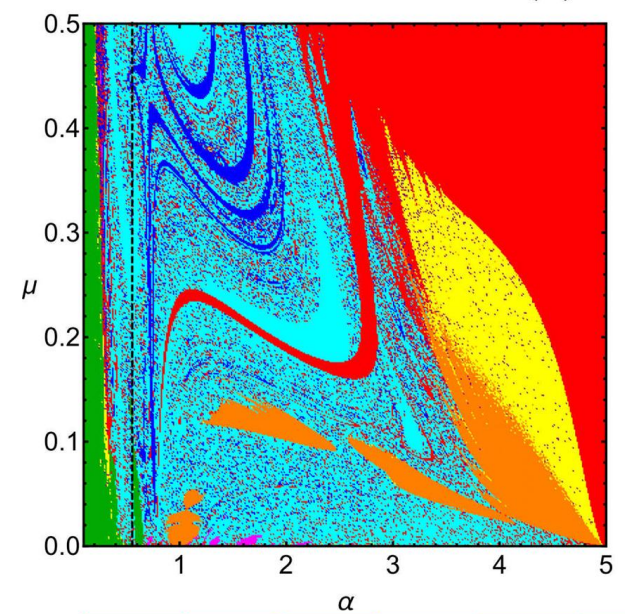

\begin{tabular}{l|l|l|l|l|l} 
Type 1a & Type 1b & Type 2a & Type 2b & Type 3a & Type 3b
\end{tabular}

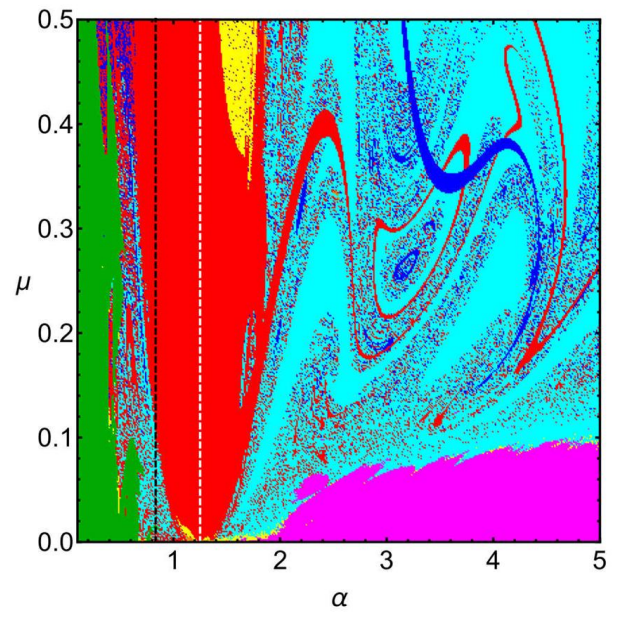

(d)

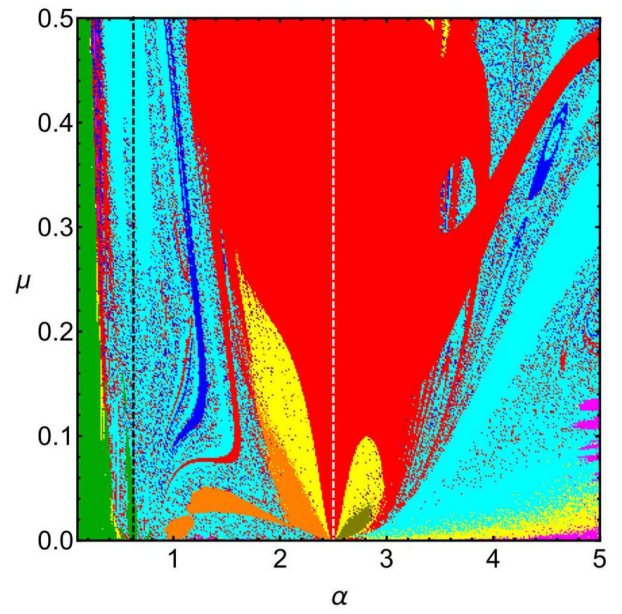

(f)

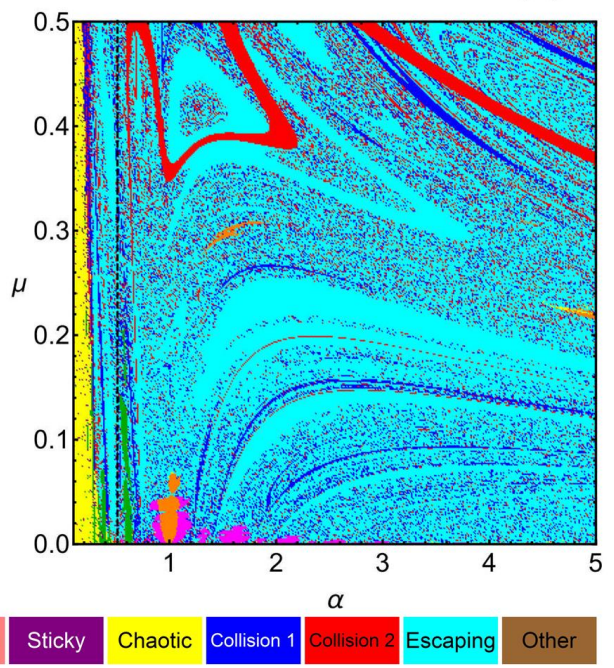

Fig. 10. Color diagrams showing the basins on the $(\alpha, \mu)$-plane, when the test particle is launched from its pericenter, for $(a): e=0,(b): e=0.2,(c): e=$ $0.4,(d): e=0.6,(e): e=0.8,(f): e=$ 0.95 . The black dashed line marks the position where the apocenter distance is $\alpha(1+e)=1$, while the white dashed line indicates the position where the pericenter distance is $\alpha(1-e)=1$.
One should expect that the dynamics on the $(\alpha, \mu)$-plane would be the same when $e=0$. However, looking at parts a of Figs. 10 and 11 it is evident that this is not true. Indeed, for $e=0$ there is no pericenter and apocenter, but according to the Eqs. (4) and (5), the test particle is launched from two different initial positions on the $x$-axis with respect to the primaries, hence the observed differences in its orbital nature.

\subsection{The $(\mu, e)$ survey}

The last type of map under consideration lies on the $(\mu, e)$-plane. In Figs. 12a-i we present the nature of the motion of the test particle when it is launched from its pericenter, for $\alpha \leq 1$. In part a, where $\alpha=0.2$, it is seen, that almost all the map is covered by starting conditions that correspond to prograde orbits around 
(a)

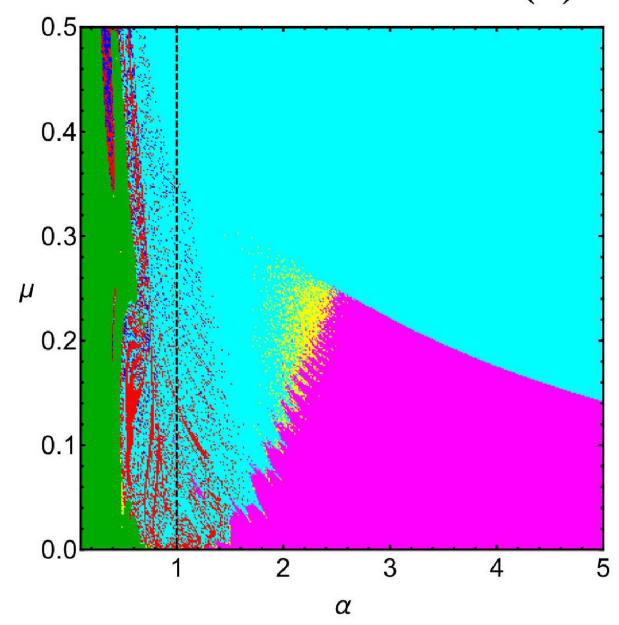

(c)

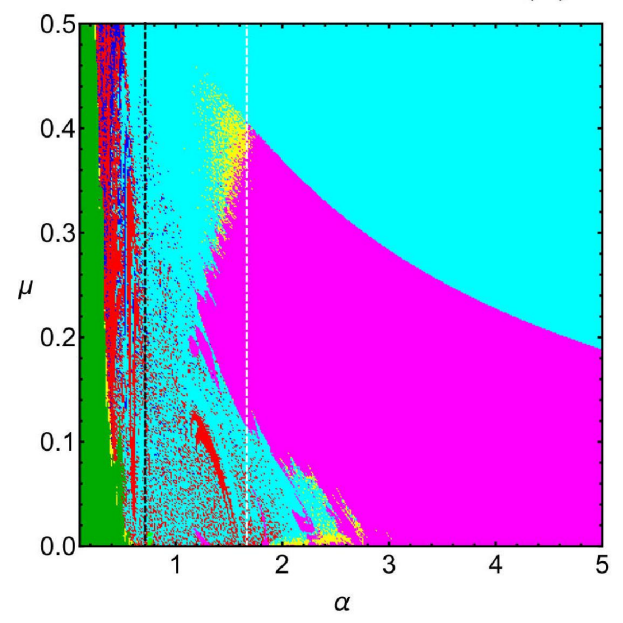

(e)

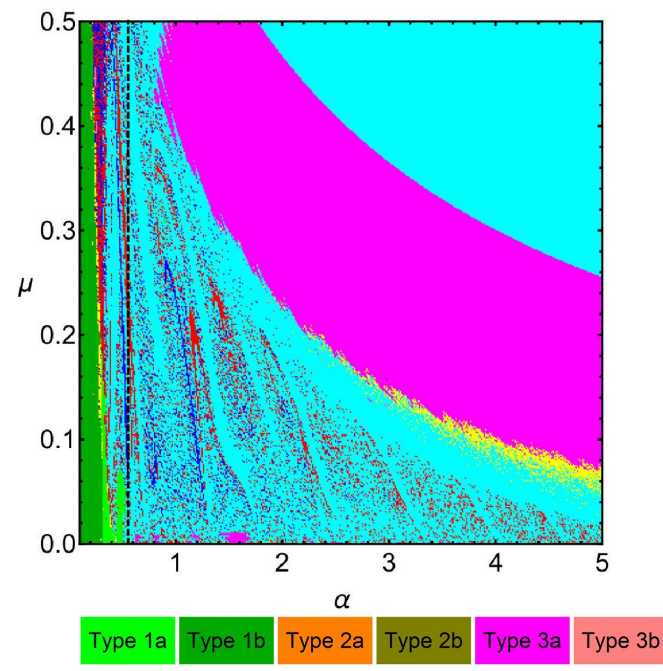

(b)

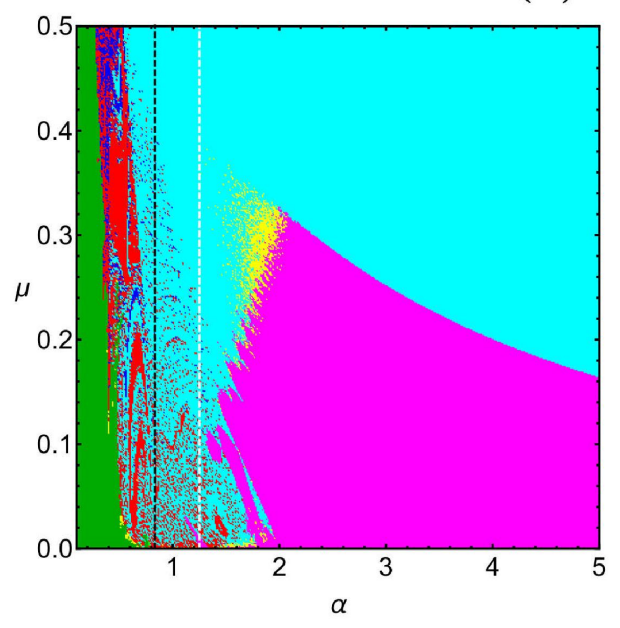

(d)

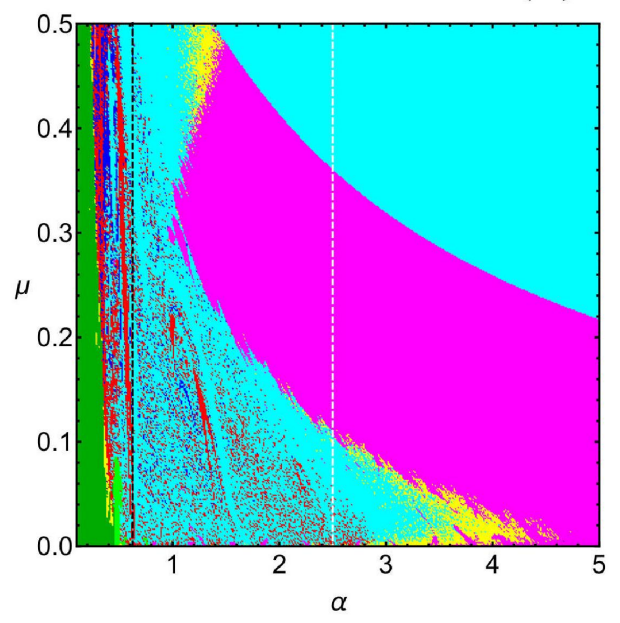

(f)

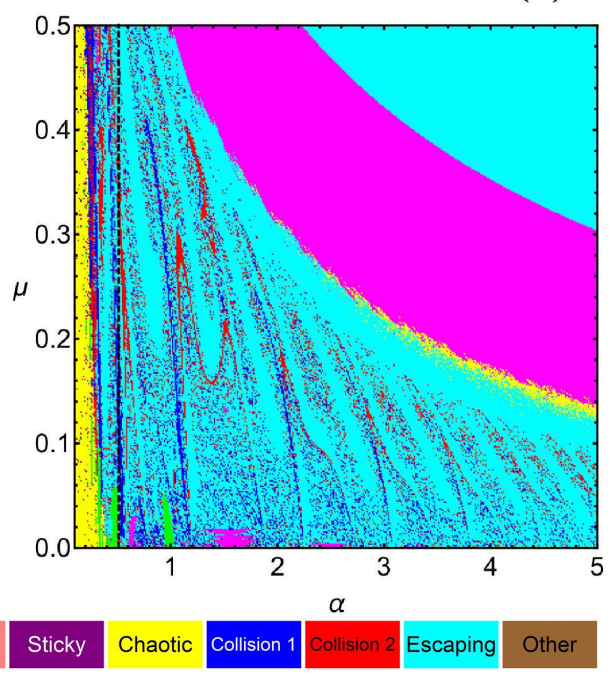

Fig. 11. Color diagrams showing the basins on the $(\alpha, \mu)$-plane, when the test particle is launched from its apocenter, for $(a)$ : $e=0,(b): e=0.2,(c): e=$ $0.4,(d): e=0.6,(e): e=0.8,(f): e=$ 0.95 . The black dashed line marks the position where the apocenter distance is $\alpha(1+e)=1$, while the white dashed line indicates the position where the pericenter distance is $\alpha(1-e)=1$. the primary. Only at extremely high values of the eccentricity $(e>0.9)$ trapped chaotic motion appears, along with collision trajectories. As the value of the semi-major axis increases (see parts $b$ and $c$ ) the portion of the prograde Type $1 b$ trajectories is reduced, while at the same time collision and escape type of motion emerge, from the right-hand side of the diagrams. When $\alpha=0.5$ (part d) and $\alpha=0.6$ (part e) the structure of the $(\mu, e)$-plane is very complicated, with a fractal-like geometry (see e.g., Aguirre et al. 2001, 2009). More specifically, the unified stability island of Type 1b motion splits into several smaller bounded basins, while the escape and collision basins form spiral structures. For $\alpha \geq 0.7$ Type $1 \mathrm{~b}$ motion is hardly visible and it is present only for low values of the mass parameter. Moreover, a basin, corresponding to collision trajectories to the secondary, 
(a)

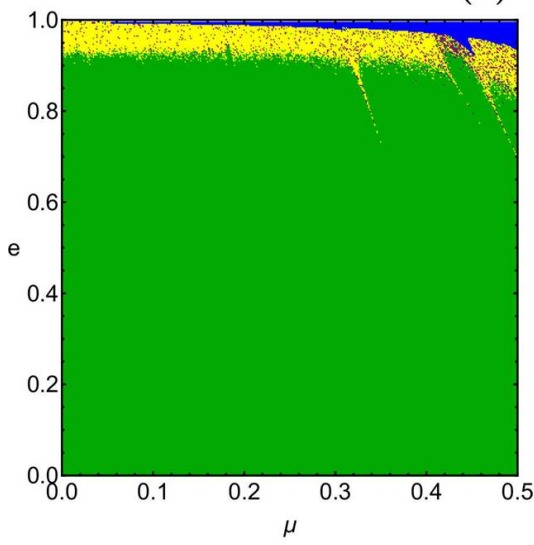

(d)

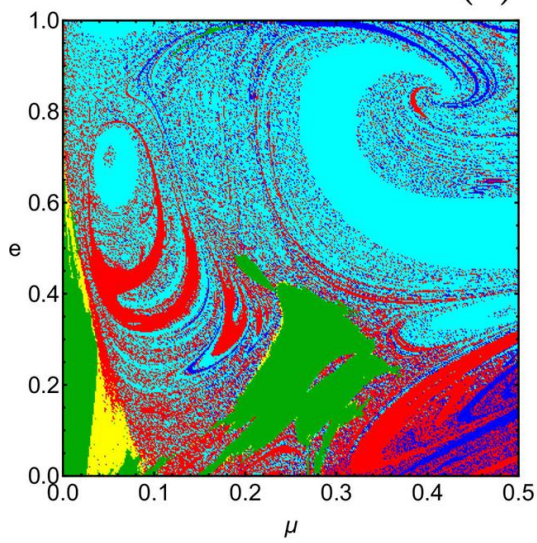

(g)

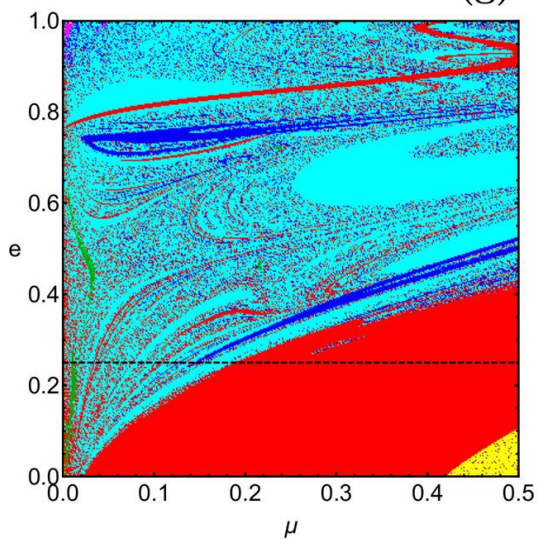

Type 1a Type 1b Type 2a Type 2b

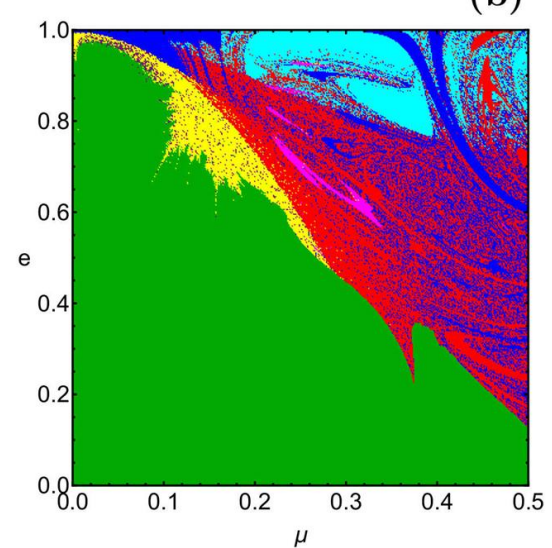

(e)

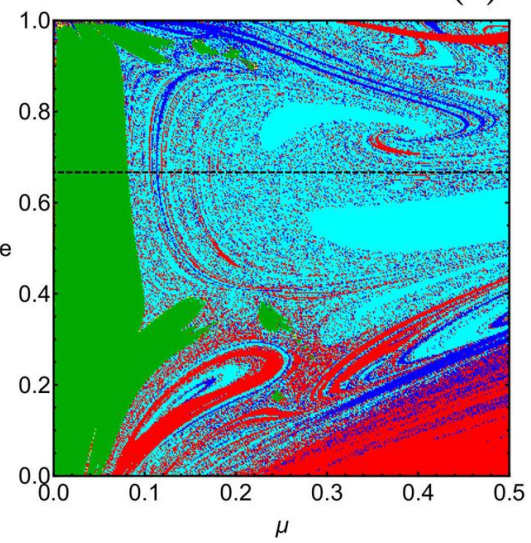

(h)

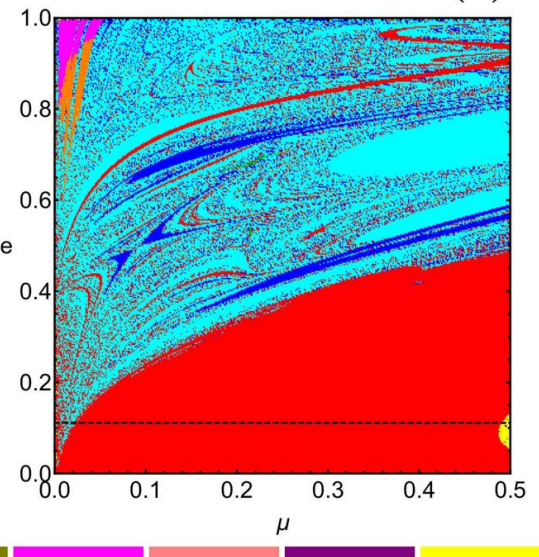

(b)

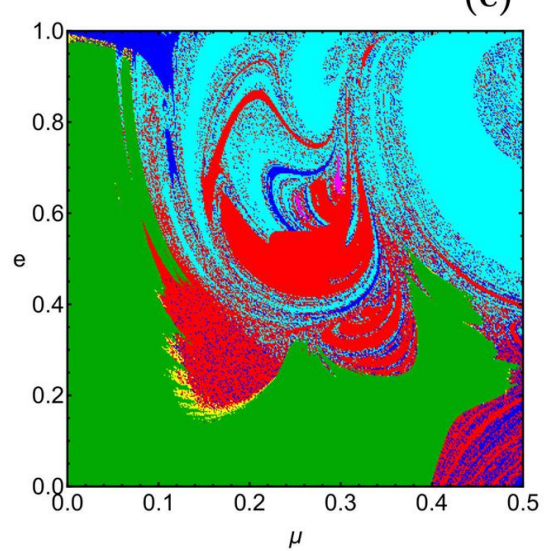

(f)

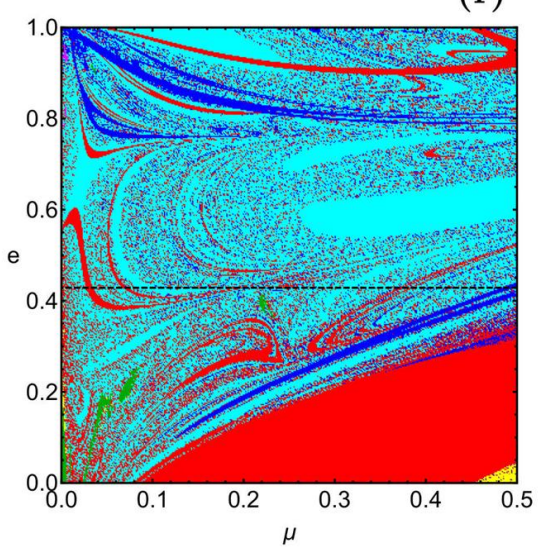

(i)

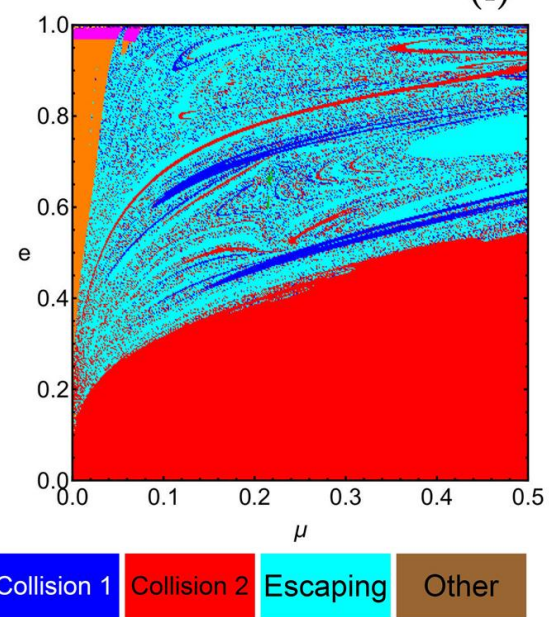

Fig. 12. Color diagrams showing the basins on the $(\mu, e)$-plane, when the test particle is launched from its pericenter, for $(a): \alpha=0.2,(b): \alpha=0.3$, $(c): \alpha=0.4,(d): \alpha=0.5,(e): \alpha=0.6,(f): \alpha=0.7,(g): \alpha=0.8,(h): \alpha=0.9,(i): \alpha=1.0$. The black dashed line marks the position where the apocenter distance is $\alpha(1+e)=1$.

establishes (at the lower right part of the diagrams) and its size grows with an increasing value of $\alpha$. For $\alpha \geq 0.9$ at the upper left corner of the $(\mu, e)$-plane we see the presence of new stability islands corresponding to retrograde motion around the secondary (Type 2a), while for higher values of the eccentricity the type of the trajectories changes to Type $3 \mathrm{a}$, thus revolving around both main bodies.

The orbital properties of the massless particle, when it is launched from its apocenter, are given in Figs. 13a-i. For relatively low values of the semi-major axis (see parts a-c) the structure of the $(\mu, e)$-plane is fairly close to what we have seen earlier in Fig. 12. The only major difference is the fact that now retrograde trajectories around the primary (Type 1a) are also possible, for high values of the eccentricity. Again, the most strange and complicated basin structures of collision trajectories occur when $0.5 \leq \alpha \leq 0.7$. For higher values of the semi-major axis Type $1 \mathrm{~b}$ motion disappears, while the strength of the collision basins weakens. In addition, at the right-hand 
(a)

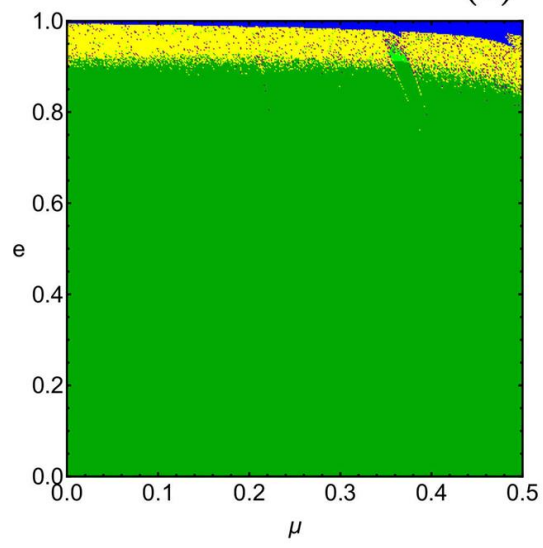

(d)

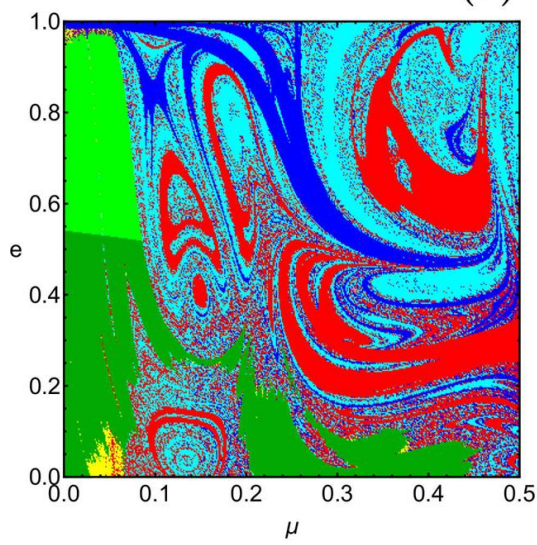

(g)

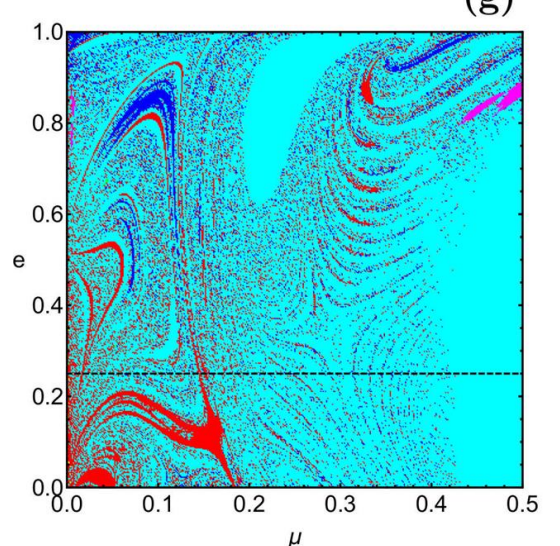

$\mu$

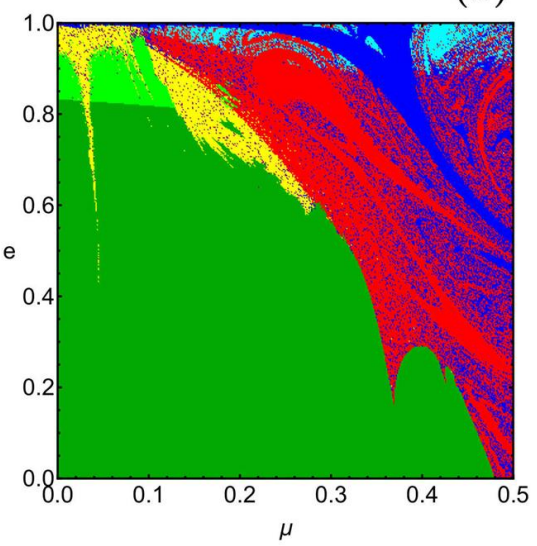

(e)

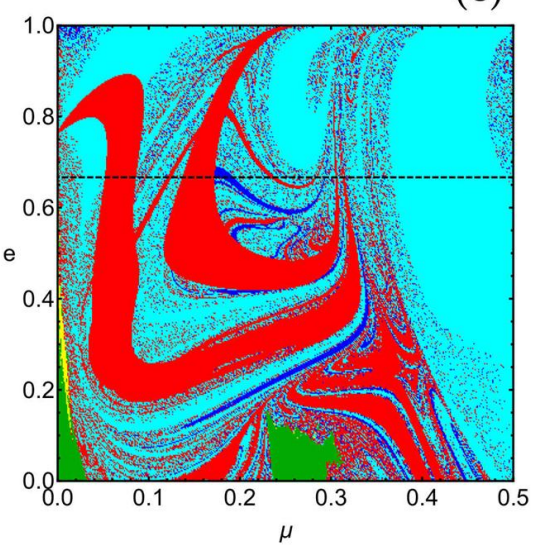

(h)

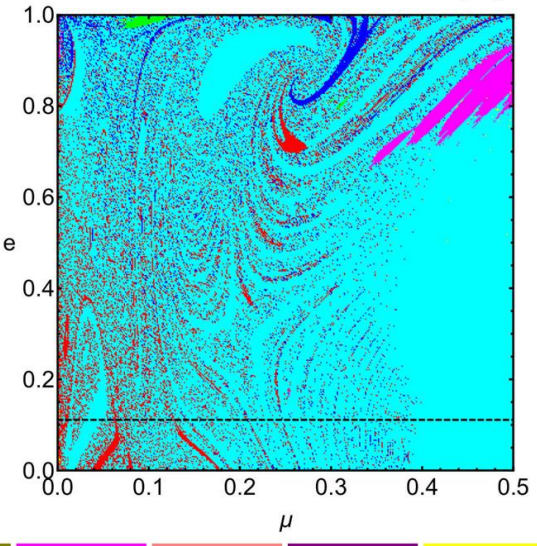

(b)

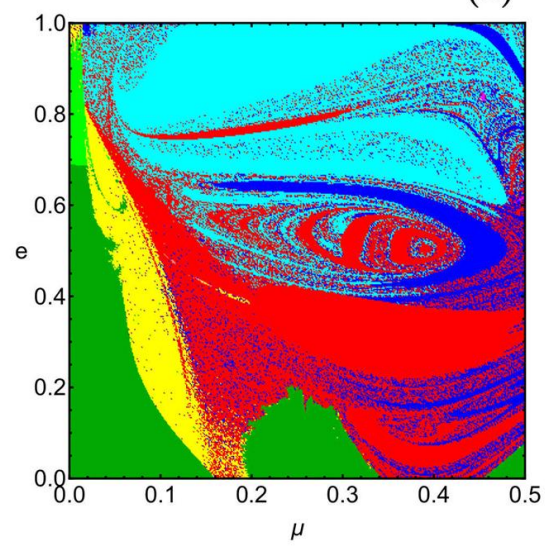

(f)

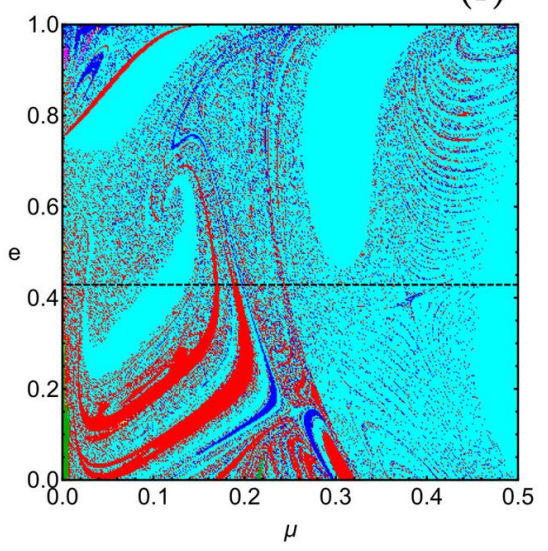

(i)

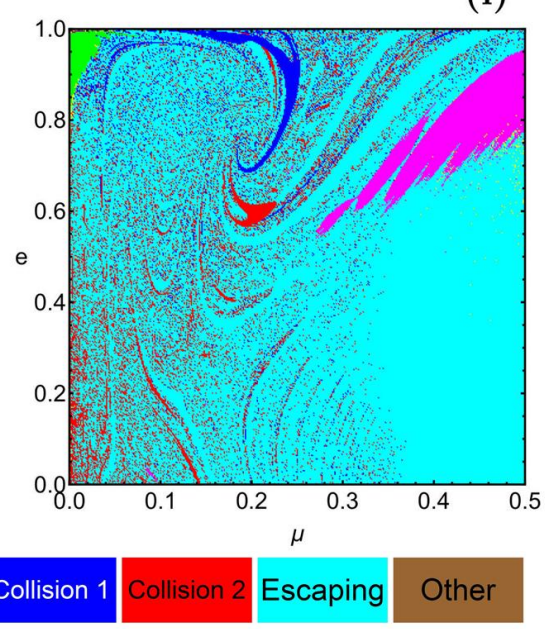

Fig. 13. Color diagrams showing the basins on the $(\mu, e)$-plane, when the test particle is launched from its apocenter, for $(a): \alpha=0.2,(b): \alpha=0.3$, $(c): \alpha=0.4,(d): \alpha=0.5,(e): \alpha=0.6,(f): \alpha=0.7,(g): \alpha=0.8,(h): \alpha=0.9,(i): \alpha=1.0$. The black dashed line marks the position where the apocenter distance is $\alpha(1+e)=1$.

side of the diagrams, we see the appearance of new stability islands, formed of starting conditions corresponding to retrograde trajectories around both main bodies (Type 3a). Also, it should be noted that when the semi-major axis of the initial eccentric orbit of the test particle tends to 1, Type 1a motion is also possible, for relatively low and high values of $\mu$ and $e$, respectively.

In Figs. 14a-d we display additional color maps on the $(\mu, e)$ plane, for values of the semi-major axis larger than 1 , when the test particle is launched from its pericenter. We see that: (i) a basin, corresponding to trajectories leading to a collision with the secondary, exists around the region, where the pericenter distance is equal to 1 . The area of this collision basin is reduced, while its relative position moves to higher values of $e$, with increasing value of the semi-major axis, (ii) at the lower-left corner of the $(\mu, e)$-plane a stability island emerges, corresponding to trajectories moving around both main bodies, in a retrograde orientation. The area of this bounded basin increases, as we 
(a)

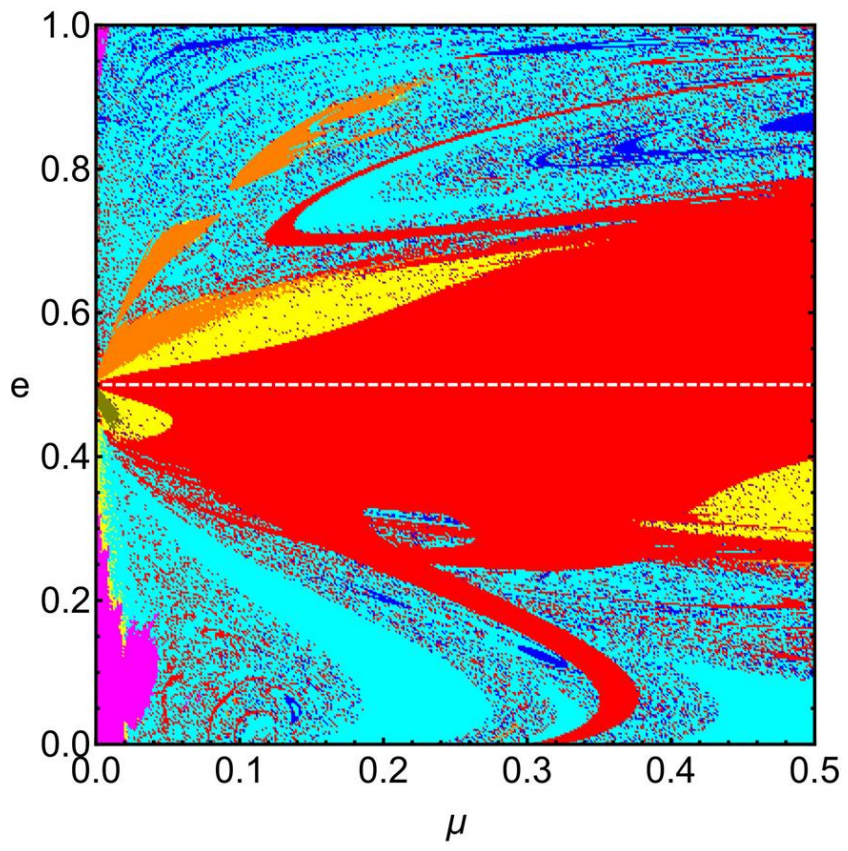

(c)

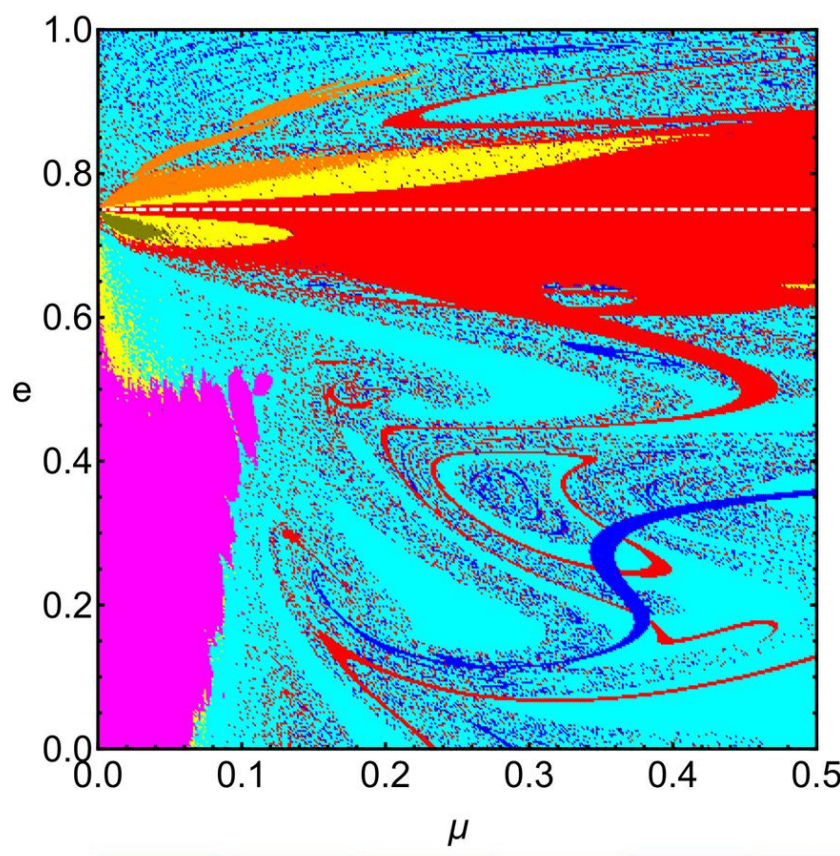

Type 1a $\quad$ Type 1b Type 2a Type 2b Type 3a Type 3b

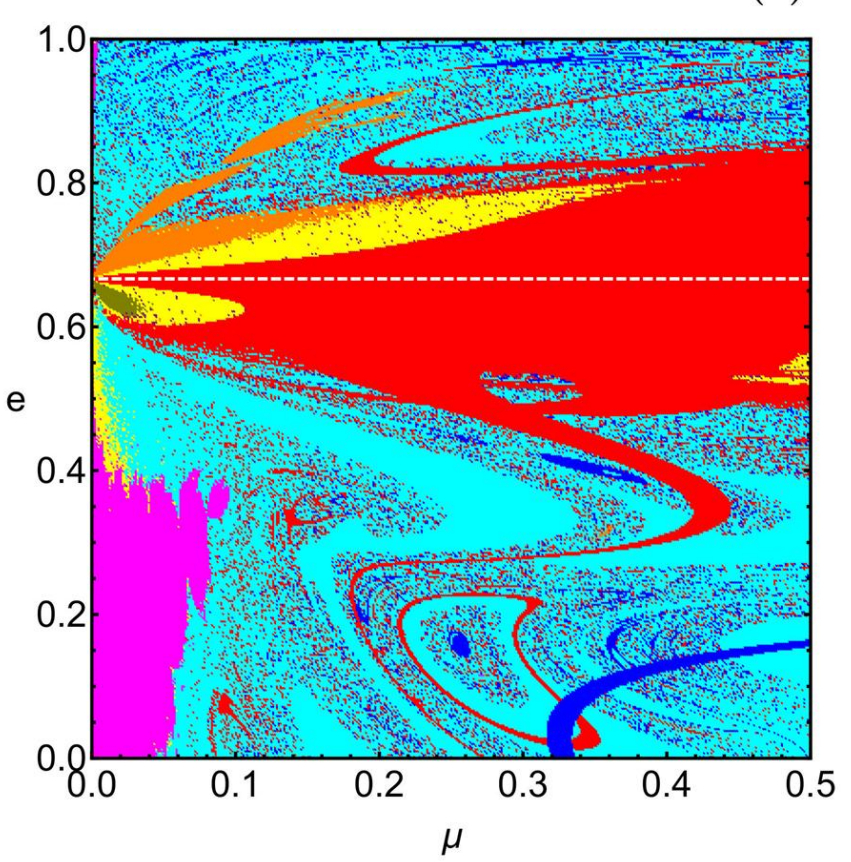

(d)

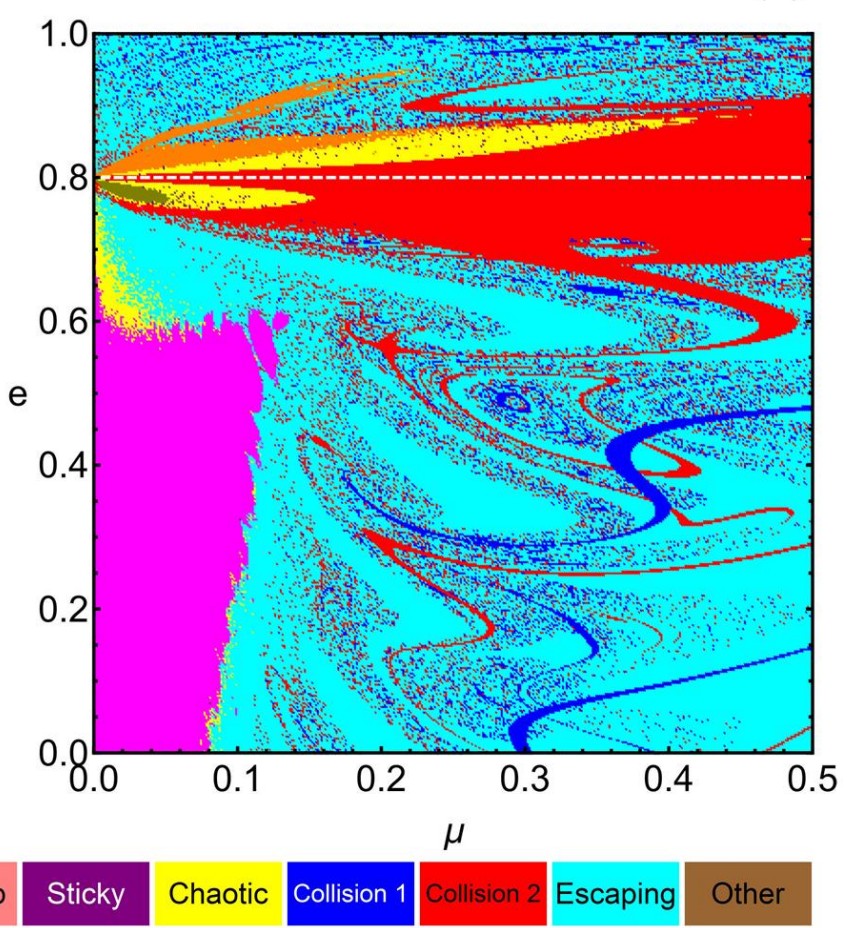

Fig. 14. Color diagrams showing the basins on the $(\mu, e)$-plane, when the test particle is launched from its pericenter, for $(a): \alpha=2,(b): \alpha=3$, $(c): \alpha=4,(d): \alpha=5$. The while the white dashed line indicates the position where the pericenter distance is $\alpha(1-e)=1$.

proceed to higher values of $\alpha$, and (iii) when $\alpha>1$ both types of ordered motion around the secondary (that is, Types $2 \mathrm{a}$ and $2 \mathrm{~b})$ are possible, with respective basins situated around the line $d_{\mathrm{p}}=1$.

In the same vein, Figs. 15a-d shows the color maps on the $(\mu, e)$-plane, for $\alpha>1$, when the test particle is launched from its apocenter. In this case, the orbital structure of the basin diagram is less interesting, with respect to those of Fig. 14. It is seen, that stability island, corresponding to Type $3 \mathrm{a}$ motion, covers a large portion of the $(\mu, e)$-plane, while its area seems to reduced, with increasing value of the semi-major axis. At the boundaries of this stability island, we have the presence of trapped chaotic motion, which is an expected phenomenon. The region below the stability islands of the Type 3 a motion is completely smooth and it is dominated by a unified basin of escape. On the other hand, the region above the same stability island is covered by a noisy mixture of escaping and collision (to both main bodies) starting conditions. 
(a)
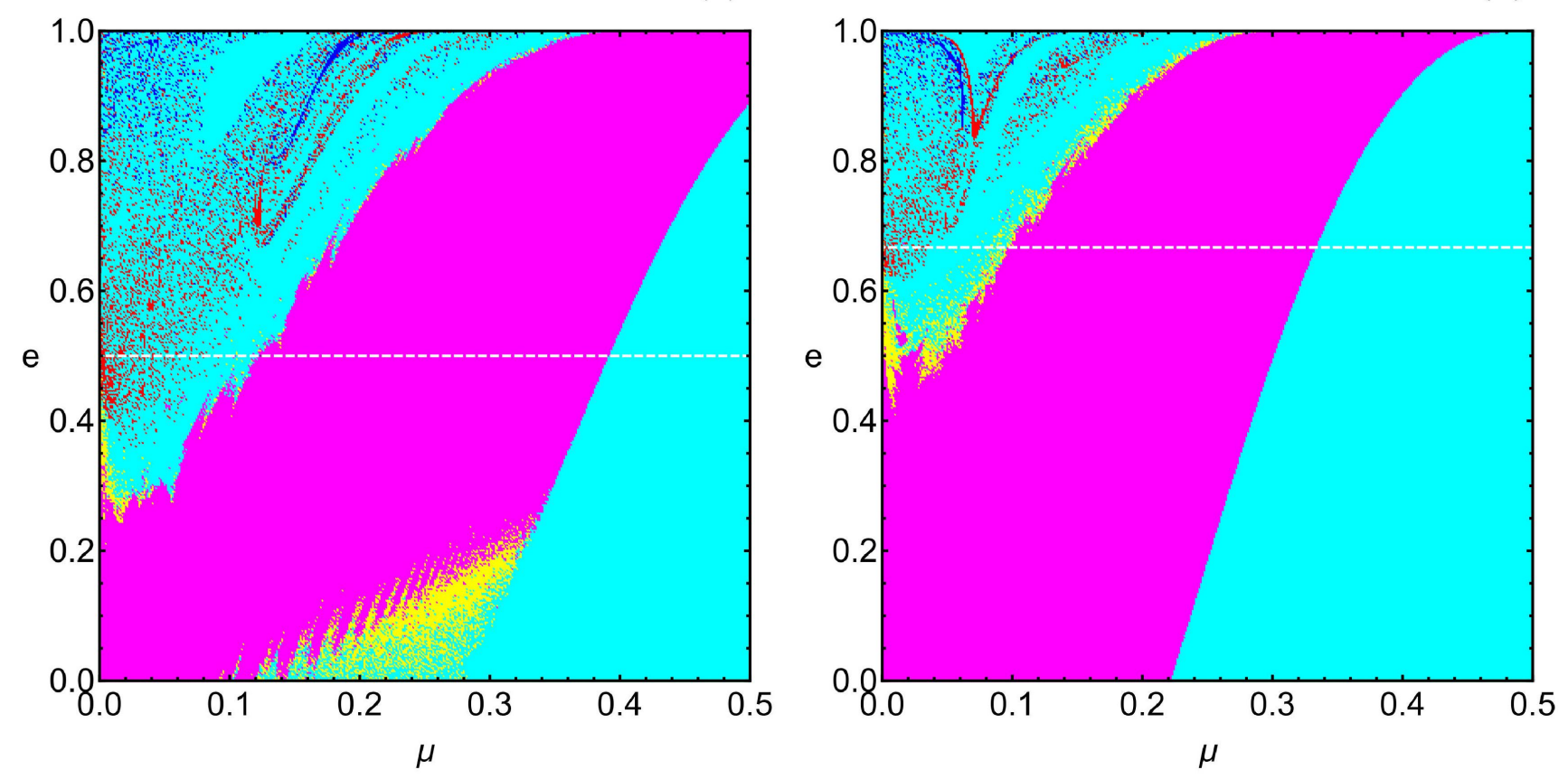

(c)

(d)
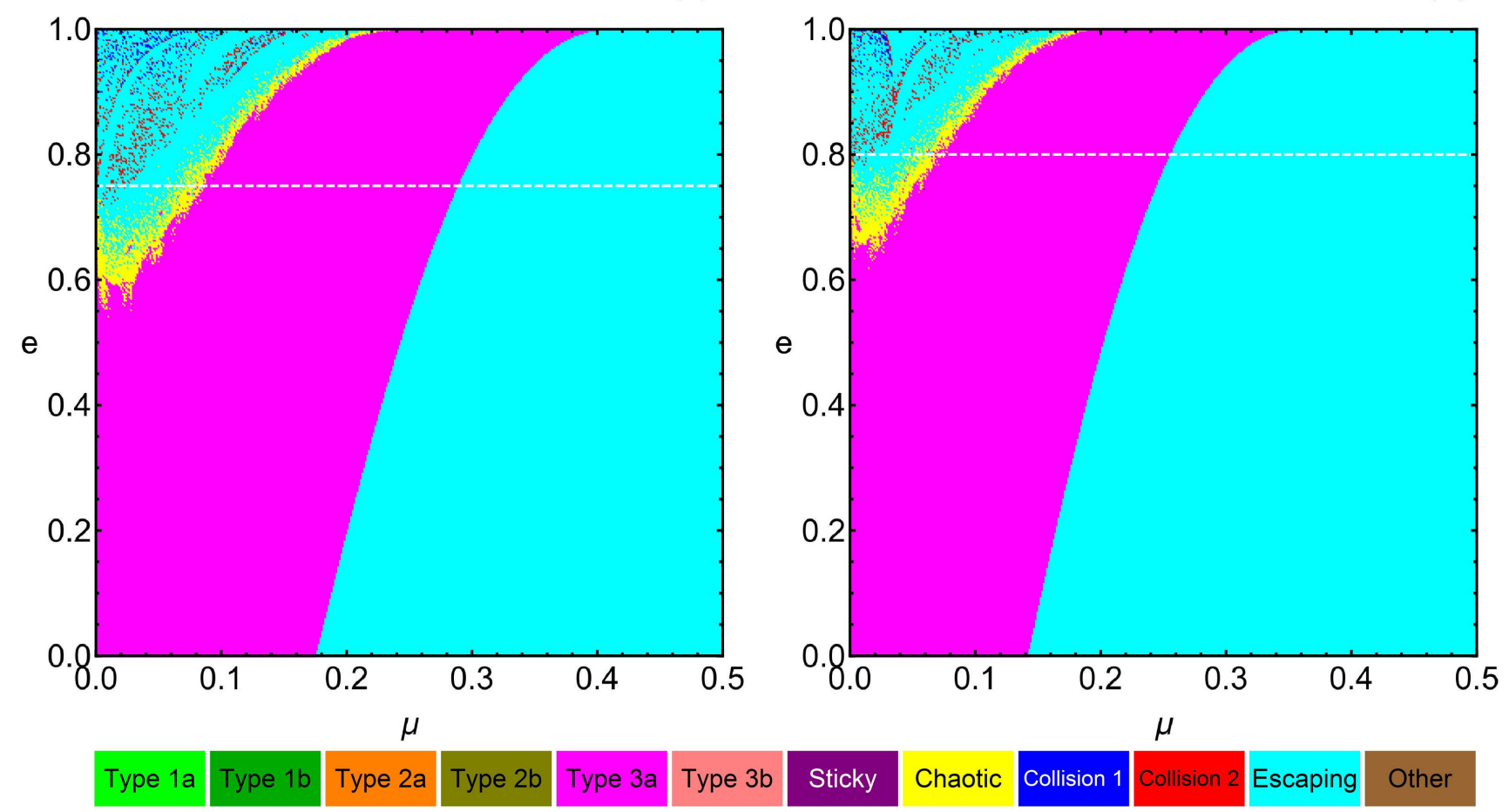

Fig. 15. Color diagrams showing the basins on the $(\mu, e)$-plane, when the test particle is launched from its apocenter, for $(a): \alpha=2,(b): \alpha=3$, $(c): \alpha=4,(d): \alpha=5$. The white dashed line indicates the position where the pericenter distance is $\alpha(1-e)=1$.

\section{Conclusions}

In this article, we combined the theory of the restricted threebody problem along with the numerical technique of grid classification, in order to predict the nature of the trajectories of a test particle moving in a binary exoplanetary system. Specifically, the binary system could be composed of a parent star along with an exoplanet, two exoplanets, or an exoplanet with an exomoon, while the third body (test particle) can be an asteroid/comet, a space probe, or even a small exomoon in the case where the primary is a star. Using the grid classification method, we managed to obtain the character of the motion of the massless particle and classify the corresponding starting conditions in three classes: (i) bounded, (ii) escaping and (iii) collision. In the case of regular bounded motion, additional criteria were used for deriving their geometry (revolving around one or both main bodies) and orientation (prograde or retrograde). For the test particle, we considered two scenarios, regarding its initial position, that is 
launched either from its pericenter or apocenter. The basin diagrams on the several types of color-coded 2D maps helped us to visualize the final states of the test particle.

Specifically, our analysis suggests that the influence of the mass parameter $\mu$, along with the semi-major axis $\alpha$ and the eccentricity $e$ of the initial eccentric trajectory of the test particle is as follows:

- Retrograde regular motion around the primary (Type 1a) exists for $\alpha<1$ and only when the test particle is launched from its apocenter.

- Prograde regular motion around the primary (Type 1b) exists for $\alpha<1$ for both pericentric and apocentric launching of the test particle.

- Bounded regular motion around the secondary (Types 2a and $2 b$ ) exists only when the massless particle starts from its pericenter. Moreover, Type 2a is present for $d_{\mathrm{p}}<1$ and Type $2 \mathrm{~b}$ for $d_{\mathrm{p}}>1$.

- Simple retrograde trajectories around both bodies (Type 3a) were found and are present for $d_{\mathrm{p}}>1$, for both pericentric and apocentric launching.

- Resonant retrograde trajectories around both bodies (Type 3a) exist for $\mu<0.1, \alpha>1, d_{\mathrm{p}}<1$, while they are possible only when the test particle is launched from its pericenter.

- Collision trajectories to secondary are mainly present around $d_{\mathrm{p}}=1$. Furthermore, the area of the respective type of basin grows, with an increasing value of $\mu$, while it reduces as the value of the semi-major axis $\alpha$ increases.

- Escaping motion seems to completely dominate for relatively high values of the mass parameter $(\mu>0.3)$ and the semimajor axis $(\alpha>3)$, in the case of apocentric launching.

- For relatively high values of the eccentricity $(e>0.9)$ the regular types of motion $1 \mathrm{a}$ and $1 \mathrm{~b}$ turn to chaotic.

- Our computations suggest, that prograde motion around both main bodies (Type $3 \mathrm{~b}$ ), along with motion (prograde and retrograde) between them (Types $4 \mathrm{a}$ and $4 \mathrm{~b}$ ) is not possible, at least for the used type of initial conditions of the test particle.

- For $0.4<\alpha<0.6$ the orbital properties of the test particle are very complicated, thus leading to highly interesting basin diagrams with a fractal-like geometry.

Additionally, it should be stated, that in exoplanetary systems that contain only one exoplanet (see Table 1) further smaller exoplanets could exist. The positions of these hidden exoplanets should be the stable regions of the systems, as they have been presented in the basin color-coded diagrams.

In closing, we would like to emphasize that the present orbit classification in exoplanetary systems is novel since there are no previous studies containing such a systematic and detailed analysis of the final states of the trajectories of the test particle. On this basis, we claim that our contribution contains new and interesting information which advances our existing knowledge regarding the orbital dynamics around exoplanets.

Acknowledgements. This project was funded by the Deanship of Scientific Research (DSR) at King Abdulaziz University, Jeddah, Saudi Arabia, under gran number (KEP-42-130-38). The authors, therefore, acknowledge with thanks DSR for technical and financial support. The first author (E.E.Z.) thanks Dr. Christof Jung for illuminating discussions, during the investigation. The authors would also like to express their warmest thanks to the anonymous referee for the careful reading of the manuscript as well as for all the apt suggestions and comments which allowed us to improve both the quality and the clarity of the paper.

\section{References}

Aguirre, J., Vallejo, J. C., \& Sanjuán, M. A. F. 2001, Phys. Rev. E, 64, 066208 Aguirre, J., Viana, R. L., \& Sanjuán, M. A. F. 2009, Rev. Mod. Phys., 81, 333

Antoniadou, K. I. 2016, Eur. Phys. J. Spec. Top., 225, 1001

Antoniadou, K. I., \& Voyatzis, G. 2014, Astrophys. Space Sci., 349, 657

Antoniadou, K. I., \& Voyatzis, G. 2016, MNRAS, 461, 3822

Campanella, G. 2011, MNRAS, 418, 1028

Campanella, G., Nelson, R. P., \& Agnor, C. B. 2013, MNRAS, 433, 3190

Correia, A. C. M., Couetdic, J., Laskar, J., et al. 2010, A\&A 511, A21

Couetdic, J., Laskar, J., Correia, A. C. M., Mayor, M., \& Udry, S. 2010, A\&A 519, A10

Érdi, B. 1977, Celest. Mech., 15, 367383

Érdi, B., \& Sándor, Zs. 2005 Celest. Mech. Dyn. Astron., 92, 113

Érdi, B., Rajnai, R., Sándor, Z., \& Forgács-Dajka, E. 2012, Celest. Mech. Dyn. Astron., 113, 95

Ferraz-Mello, S., Michtchenko, T. A., \& Beaugé, C. 2006, Chaotic Worlds: from Order to Disorder in Gravitational N-Body Dynamical Systems, eds. B. A. Steves, A. J. Maciejewski, \& M. Hendry (Berlin: Springer), 255

Funk, B., Schwarz, R., Süli, A., \& Érdi, B. 2012, MNRAS, 423, 3074

Goździewski, K., Konacki, M., \& Maciejewski, A. J. 2006, ApJ, 645, 688

Hadjidemetriou, J. D. 2006, Celest. Mech. Dyn. Astron., 95, 225

Henrard, J., \& Libert, A.-S. 2008, Celest. Mech. Dyn. Astron., 102, 177

Hippke, M., \& Angerhausen, D. 2015, ApJ, 811, 5

Laskar, L., \& Correia, A. C. M. 2009, A\&A, 496, L5

Laughlin, G., \& Chambers, J. E. 2002, AJ, 124, 592

Lee, M. H. 2004, ApJ, 611, 517

Leleu, A., Robutel, P., Correia, A. C. M., \& Lillo-Box, J. 2017, A\&A, 599, A4

Lovis, C., Ségransan, D., Mayor, M., et al. 2011, A\&A, 528, A112

Michtchenko, T. A., Beaugé, C., \& Ferraz-Mello, S. 2006, Celest. Mech. Dyn. Astron., 94, 411

Mikkola, S., Innanen, K., Wiegert, P., Connors, M., \& Brasser, R. 2006 , MNRAS, 369, 15

Morais, M. H. M., \& Namouni, F. 2013, Celest. Mech. Dyn. Astron., 117, 405

Murray, C., \& Dermott, S. 1999, Solar System Dynamics (Cambridge: Cambridge Univesristy Press)

Nagler, J. 2004, Phys. Rev. E, 69, 066218

Nagler, J. 2005, Phys. Rev. E, 71, 026227

Namouni, F. 1999, Icarus, 137, 293

Pousse, A., Robutel, P., \& Vienne, A. 2017, Celest. Mech. Dyn. Astron., 128, 383

Press, H. P., Teukolsky, S. A, Vetterling, W. T., \& Flannery, B. P. 1992, Numerical Recipes in FORTRAN, 2nd edn., (Cambridge: Cambridge Univ. Press), 77

Rein, H., Papaloizou, J. C. B., \& Kley, W. 2010, A\&A, 510, A4

Robutel, P., \& Pousse, A. 2013, Celest. Mech. Dyn. Astron., 117, 17

Schwarz, R., Bazso, A., Érdi, B., \& Funk, B. 2012, MNRAS, 427, 397

Sidorenko, V., Artemiev, A., Neishtadt, A., \& Zelenyi, L. 2014, Celest. Mech. Dyn. Astron., 120, 131

Skokos, C. 2001, J. Phys. A Math. Gen, 34, 10029

Teachey, A., \& Kipping, D. M. 2018, Sci. Adv., 4, 1784

Voyatzis, G. 2008, ApJ, 675, 802

Wolfram, S. 2003, The Mathematica Book Champaign: Wolfram Media 